\title{
Glutamine reliance in cell metabolism
}

\author{
Hee Chan Yoo', Ya Chun Yu', Yulseung Sung ${ }^{1}$ and Jung Min Han ${ }^{1,2}$
}

\begin{abstract}
As knowledge of cell metabolism has advanced, glutamine has been considered an important amino acid that supplies carbon and nitrogen to fuel biosynthesis. A recent study provided a new perspective on mitochondrial glutamine metabolism, offering mechanistic insights into metabolic adaptation during tumor hypoxia, the emergence of drug resistance, and glutaminolysis-induced metabolic reprogramming and presenting metabolic strategies to target glutamine metabolism in cancer cells. In this review, we introduce the various biosynthetic and bioenergetic roles of glutamine based on the compartmentalization of glutamine metabolism to explain why cells exhibit metabolic reliance on glutamine. Additionally, we examined whether glutamine derivatives contribute to epigenetic regulation associated with tumorigenesis. In addition, in discussing glutamine transporters, we propose a metabolic target for therapeutic intervention in cancer.
\end{abstract}

\section{Introduction}

After Otto Warburg discovered that cancer cells exhibit significantly elevated glucose consumption and lactate secretion even in the presence of oxygen ${ }^{1}$, studies on cell metabolism have accumulated. The major findings are that aerobic glycolysis is not a symptom of impaired mitochondrial function, and that glutamine supports mitochondrial oxidative metabolism when pyruvate derived from glucose is converted into lactate and secreted $^{2-4}$. Glutamine, which is a nonessential amino acid (NEAA) due to the endogenous glutamine biosynthesis pathway, is currently considered essential in cancer cells because transformed cells consume glutamine at a rate exceeding that of glutamine biosynthesis ${ }^{5}$. Glutamine has a versatile role in cell metabolism, participating in tricarboxylic acid (TCA) cycle supplementation and the biosynthesis of nucleotides, glutathione (GSH), and other nonessential amino acids. Thus, glutamine deprivation suppresses cancer growth and even induces cell death in several cancers ${ }^{6,7}$. This metabolic dependency of

Correspondence: Jung Min Han (jhan74@yonsei.ac.kr)

${ }^{1}$ Yonsei Institute of Pharmaceutical Sciences, College of Pharmacy, Yonsei University, Incheon 21983, South Korea

2Department of Integrated OMICS for Biomedical Science, Yonsei University, Seoul 03722, South Korea

These authors contributed equally: Hee Chan Yoo, Ya Chun Yu, Yulseung Sung transformed cells on glutamine constitutes the recently defined glutamine addiction ${ }^{8}$.

Since glutaminase 1 (GLS1), a key mitochondrial enzyme that catalyzes the deamidation of glutamine, was first discovered in the kidney in $1958^{9}$, many enzymes involved in glutamine metabolism have been reported ${ }^{4}$. In addition, glutamine has been confirmed to be a major nutrient source for oxidative metabolism in some cancer cell lines ${ }^{10-12}$, and specific genetic interference with glutaminase (GLS) inhibits tumor cell growth ${ }^{13}$. Moreover, CB-839, the first glutaminase inhibitor, has entered several clinical trials ${ }^{14,15}$. Despite the importance of mitochondrial glutamine metabolism, the mitochondrial glutamine transporter, encoded by a transcript variant of the SLC1A5 gene, which encodes a well-known plasma membrane glutamine transporter, was only recently discovered $^{16}$. Thus, glutamine metabolism is intriguingly linked with intricate cell metabolic processes via enzymes associated with mitochondrial glutaminolysis, cytosolic glutamine metabolism, and glutamine-derived metabolites that perform diverse cellular functions.

In this review, we first introduce metabolic pathways that enable glutamine to respond to diverse cellular needs and then discuss the metabolic link by which glutaminederived metabolites may affect cellular metabolic processes, including NEAA synthesis, epigenetic modifications, and hypoxia adaptation. We next discuss recent 
advances in glutamine metabolism with particular emphasis on tumorigenesis. We aim to offer both the principles underlying cellular dependence on glutamine metabolism under various conditions and a discussion of future directions that are leading our efforts to investigate the role of glutamine in cellular metabolism.

\section{Glutamine metabolic pathways}

Glutamine is transported into cells through plasma membrane glutamine transporters such as SLC1A5, SLC38A1, and SLC38A2 ${ }^{17}$ and can then be used for the biosynthesis of hexosamine, nucleotides, and asparagine in the cytoplasm (Fig. 1). For mitochondrial glutaminolysis, cytosolic glutamine must be transported through the inner mitochondrial membrane via the SLC1A5 variant, a mitochondrial glutamine transporter ${ }^{16}$. Next, glutamine is converted into glutamate by GLSs, amidohydrolase enzymes that catalyze the conversion of glutamine into glutamate, releasing ammonium ions. GLSs have at least three isoforms, GLS1, GLS2, and GAC (a splicing isoform of GLS1), all of which were recently reported to be localized in mitochondria ${ }^{18-20}$. Mitochondrial glutamate generated via these catabolic pathways can be exported from mitochondria to the cytosol through the SLC25A18 and SLC25A22 transporters ${ }^{21}$, and cytosolic glutamate then participates in the biosynthesis of glutathione-a tripeptide comprising glutamate, cysteine, and glycineand NEAAs (alanine, proline, aspartate, asparagine, and arginine) and is used as an exchange factor for importing extracellular cystine via SLC7A11. Mitochondrial glutamate is subsequently converted into alpha-ketoglutarate $(\alpha-K G)$ by glutamate dehydrogenase 1 (GLUD1 or GDH1) or by several mitochondrial aminotransferases, including glutamic-pyruvic transaminase 2 (GPT2) and glutamicoxaloacetic transaminase 2 (GOT2). In addition, $\alpha-\mathrm{KG}$ is exported from mitochondria through SLC25A11 to the cytosol $^{21}$ and then participates in fatty acid biosynthesis and NADH generation ${ }^{22}$ (Fig. 1). Mitochondrial $\alpha-K G$ can then participate in the TCA cycle, supporting the oxidative phosphorylation (OXPHOS) pathway or the reductive carboxylation pathway ${ }^{23}$. In the oxidative phosphorylation pathway, metabolites of glutamine participate in the generation of an electron donor, such as NADH or $\mathrm{FADH}_{2}$, after synthesis of guanosine triphosphate (GTP) and adenosine triphosphate (ATP). In addition to pyruvate-derived acetyl-CoA, $\alpha$-KG-derived metabolites (e.g., succinate and fumarate) generated via glutaminolysis are considered oncometabolites contributing to tumorigenesis $^{23}$. Citrate, generated by reductive carboxylation of $\alpha-K G$, is especially crucial for lipid synthesis under lowoxygen conditions ${ }^{24,25}$.

$\alpha-K G$ is considered an important cofactor for enzymes participating in epigenetic modification ${ }^{26}$. It is a substrate for $\alpha$-ketoglutarate dehydrogenase (OGDH) in oxidative reactions generating succinyl-CoA and isocitrate dehydrogenase 1 (IDH1) or isocitrate dehydrogenase 2 (IDH2), which catalyze the reductive carboxylation reaction converting $\alpha-K G$ to isocitrate. Cancer cells in tumors with IDH1 or IDH2 mutations show oncogenic activity by converting glutamine-derived $\alpha-K G$ to 2 -hydroxyglutarate (2-HG), which competitively inhibits $\alpha$-KG-dependent histone and DNA modification enzymes ${ }^{27}$. Additionally, glutamine-derived aspartate plays a crucial role in hypoxic environments or environments with electron transport chain (ETC) impairment ${ }^{28}$. In addition, NADPH generation via glutamine metabolism in cancer cells supports redox homeostasis by maintaining the cytosolic NADPH pool used to restore oxidized glutathione ${ }^{29}$ (Fig. 1).

\section{Nucleotides synthesized from glutamine}

Cytosolic glutamine supports nucleotide biosynthesis, which is essential for rapidly proliferating cells ${ }^{30}$. The gamma-nitrogen of glutamine is used in five reactions in de novo nucleotide synthesis, and its bioavailability controls de novo biosynthesis of pyrimidines and purines (Fig. 2$)^{5}$. In purine biosynthesis, two glutamines are used to generate inosine monophosphate (IMP), a precursor of both adenosine monophosphate (AMP) and guanosine monophosphate (GMP). Then, one glutamine molecule is needed for the conversion of IMP to $\mathrm{GMP}^{31}$. In pyrimidine biosynthesis, one glutamine molecule is consumed by a carbamoyl phosphate synthetase enzyme (CPS1 or CPS2, which are localized in the mitochondria and cytosol, respectively). One more glutamine molecule is used to synthesize cytidine triphosphate (CTP) from uridine triphosphate (UTP) ${ }^{31}$. Interestingly, the first step in de novo pyrimidine biosynthesis mediated by CPSs occurs mainly in mitochondria via CPS1 in K-Ras/LKB1mutant lung cancer cells ${ }^{32}$ (Fig. 2). Although cytosolic CPS2 can produce a cytosolic pool of carbamoyl phosphate, CPS1 is a major rate-limiting enzyme in pyrimidine biosynthesis using nitrogen released via mitochondrial glutaminolysis ${ }^{32}$.

In addition, glutamine can support nucleotide synthesis via other pathways. Aspartate, which is derived from the transamination of glutamine to form glutamate, participates in pyrimidine and purine biosynthesis ${ }^{28}$. Thus, exogenous aspartate can restore cell cycle arrest caused by glutamine deprivation ${ }^{33}$. Moreover, glutamine-induced activation of mTORC1 results in the phosphorylation of the enzyme complex called carbamoyl phosphate synthetase 2, aspartate transcarbamylase, and dihydroorotase (CAD), which catalyzes the condensation reaction converting glutamine-derived nitrogen into the pyrimidine precursor orotate ${ }^{34,35}$. Notably, increased expression of the transcription factor MYC, which is strongly associated with glutamine metabolism, induces the expression of several key enzymes in nucleotide biosynthesis, 


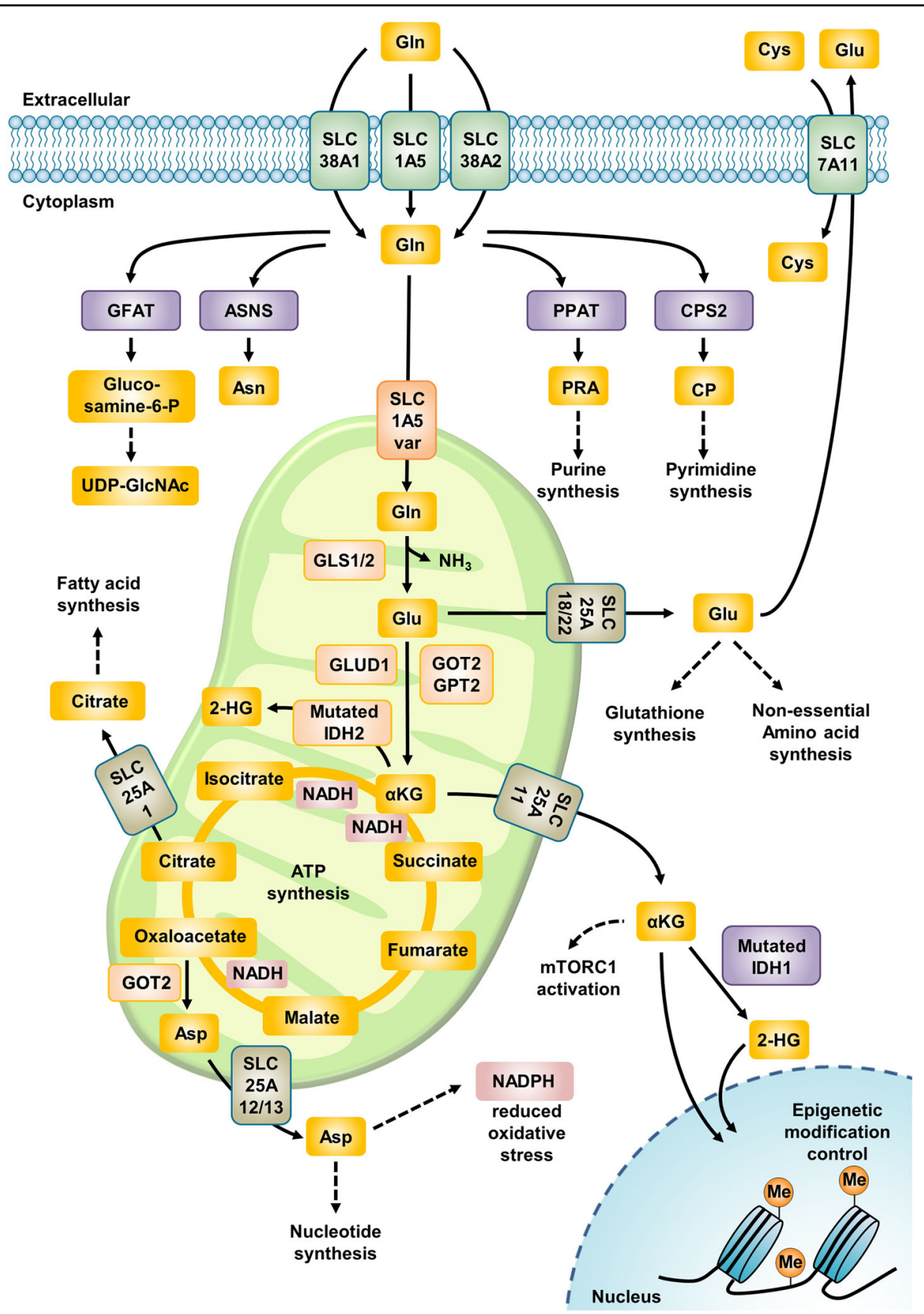

Fig. 1 Glutamine metabolic pathways. Glutamine enters through several plasma membrane glutamine transporters and is then utilized in the cytosol in processes such as the biosynthesis of nucleotides, asparagine, and UDP-GICNAc. For glutaminolysis, glutamine is transported into the mitochondrial matrix through the SLC1A5 variant and subsequently converted to glutamate by GLS. Next, GLUD1 or several aminotransferases catalyze the deamidation of glutamate, producing a-KG. Glutamine-derived a-KG supplies metabolites for the TCA cycle and fuels the generation of 2-HG under conditions of IDH2 mutation or hypoxia. Citrate derived from glutamine via reductive carboxylation supports fatty acid synthesis under conditions of hypoxia or HIF-2a transcription factor stabilization. Glutamine-derived a-KG also activates the mTORC1 pathway. A-KG and 2-HG affect epigenetic modification through a-KG-dependent dioxygenases. Gln glutamine, Glu glutamate, Asn asparagine, Cys cystine, Asp aspartate, aKG aketoglutarate, PRA 5-phosphoribosyl-1-amine, CP carbamoyl phosphate, GFAT glutamine-fructose-6-phosphate transaminase, ASNS asparagine synthetase, PPAT phosphoribosyl pyrophosphate amidotransferase, CPS carbamoyl phosphate synthetase, GLS glutaminase, GLUD glutamate dehydrogenase, GOT glutamic-oxaloacetic transaminase, GPT glutamic-pyruvate transaminase, IDH isocitrate dehydrogenase, 2-HG 2hydroxyglutarate, Me methylation. 


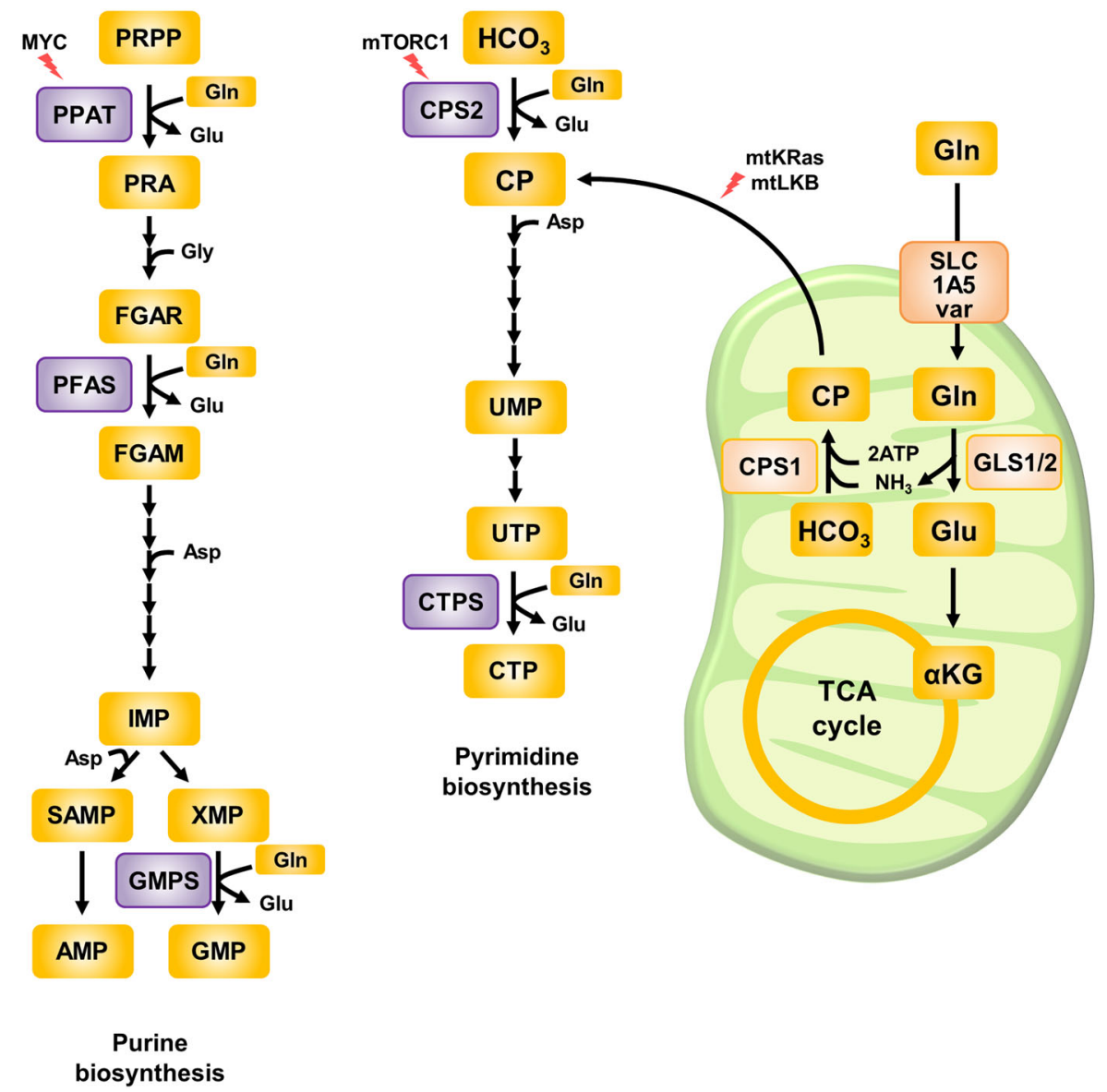

Fig. 2 Nucleotide biosynthesis from glutamine. In purine biosynthesis, two glutamine molecules are consumed to synthesize AMP, and three glutamine molecules are used to synthesize GMP. Similarly, in pyrimidine biosynthesis, one glutamine molecule is consumed to synthesize UMP, and two glutamine molecules are spent to convert UTP into CTP. The initial step in de novo pyrimidine synthesis is the condensation reaction between glutamine and bicarbonate catalyzed by CPS to produce CP. In cells with an oncogenic mutational status, including K-Ras mutation, glutaminolysis sustains mitochondrial generation of CP by providing enough nitrogen fuel as ammonium ions, and mitochondrial CP then participates in cytosolic de novo pyrimidine synthesis. Glutamine-induced nucleotide biosynthesis is also enhanced by MYC or growth signals such as mTORC1 activation PPAT phosphoribosyl pyrophosphate amidotransferase, PFAS phosphoribosylformylglycinamidine synthase, GMPS GMP synthetase, CPS carbamoyl phosphate synthetase, CTPS CTP synthetase, GLS glutaminase, PRPP 5-phosphoribosyl-1-pyrophosphate, PRA 5-phosphoribosyl-1-amine, FGAR N2formyl-N1-(5-phospho-D-ribosyl)glycinamide, FGAM 2-(formamido)-N1-(5-phospho-D-ribosyl)acetamidine, IMP inosine monophosphate, SAMP adenylosuccinate, XMP xanthosine monophosphate, AMP adenosine monophosphate, GMP guanosine monophosphate, CP carbamoyl phosphate, UMP uridine monophosphate, UTP uridine triphosphate, CTP cytidine, Glu glutamine, Glu glutamate, aKG a-ketoglutarate.

including phosphoribosyl pyrophosphate amidotransferase $(\text { PPAT })^{36}$. PPAT transfers glutamine-derived nitrogen to 5-phosphoribosyl pyrophosphate (PRPP), and this step is considered the initial step in purine biosynthesis ${ }^{37}$. In pancreatic cancer cells, oncogenic K-Ras maintains the nucleotide pool via the MAPK-dependent signaling pathway, leading to MYC upregulation, and the use of MEK inhibitors reduces the incorporation of glutaminederived nitrogen into purine nucleotides ${ }^{38}$. Collectively, these studies describe a mechanism by which glutaminederived nitrogen is essential for the rapid proliferation of cancer cells corresponding to an urgent need for nucleotide biosynthesis.

\section{NEAAs synthesized from glutamine}

Although glutamine has been considered an NEAA that is synthesized endogenously, most cancer cells cannot proliferate or survive in a medium that does not contain glutamine $e^{5}$. This inability is probably due to the function of glutamine metabolism, which provides both carbon and nitrogen for cellular biogenesis. Glutamine-derived carbon is an important substrate that supports the TCA cycle and the synthesis of glutathione. In addition, nitrogen derived from glutamine is required for the biosynthesis of molecules such as nucleotides, glucosamine, and NEAAs ${ }^{39}$. Notably, among NEAAs, the generation of glutamate and asparagine is directly dependent on glutamine (Fig. 3). 


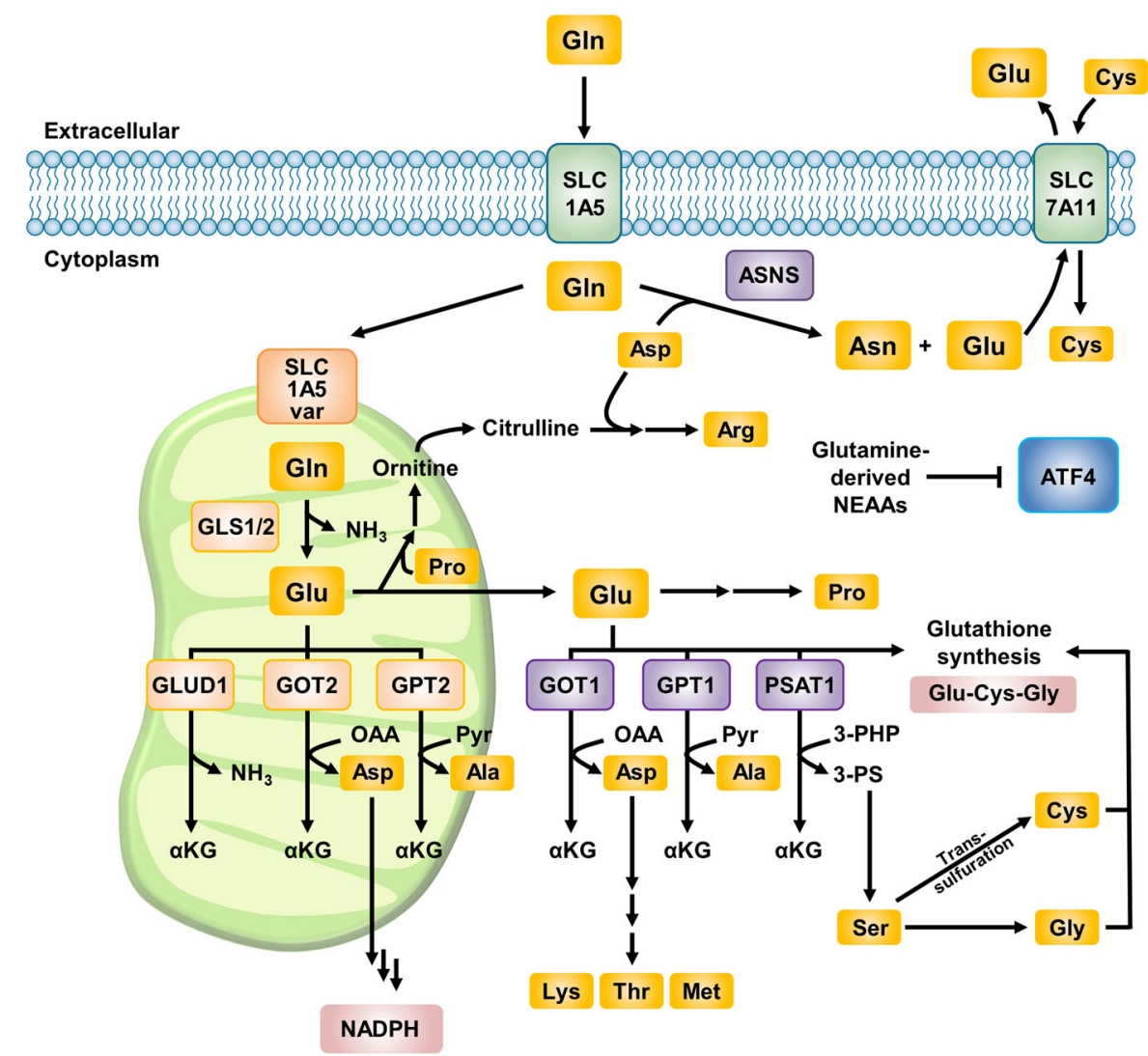

Fig. 3 NEAAs synthesized from glutamine. Intracellular glutamine is converted into diverse NEAAs and supports protein translation and amino acid signaling. Glutamine-derived glutamate plays a central role as a substrate for several aminotransferases producing aspartate, alanine, proline, arginine, serine, cysteine, and glycine. ASNS directly utilizes cytosolic glutamine to synthesize Asn, which plays a distinct role in glutamine-related metabolism. Collectively, glutamine-derived NEAAs suppress ATF4, which is a master transcriptional regulator stimulated under stress conditions. NEAAs nonessential amino acids, GLS glutaminase, GLUD glutamate dehydrogenase, GOT glutamic-oxaloacetic transaminase, GPT glutamic-pyruvate transaminase, PSAT phosphoserine aminotransferase, ATF activating transcription factor, ASNS asparagine synthetase, Gln glutamine, Glu glutamate, Pro proline, Asp aspartate, Ala alanine, Ser serine, Gly glycine, Cys cystine, Asn asparagine, Lys lysine, Thr threonine, Met methionine, aKG aketoglutarate, OAA oxaloacetate, Pyr pyruvate, PHP phosphohydroxypyruvate, PS phosphoserine.

\section{Glutamate}

Glutamate plays a central role in NEAA metabolism because it is crucial for the biosynthesis of alanine, aspartate, proline, and serine, which are in turn used for the biosynthesis of asparagine, arginine, cysteine, and glycine (Fig. 3). Glutamate is converted to $\alpha-K G$ both via GLUD1, generating glutamate-derived nitrogen as ammonia, and via aminotransferases, which transfer nitrogen from glutamate to $\alpha-K G$ to produce other NEAAs. Glutamate consumption by aminotransferases to generate NEAAs has also been indicated to be required for tumor growth in diverse cancer types ${ }^{29,40-42}$.

Although glutamate is the major downstream product of glutamine, glutamate supplementation during glutamine deprivation cannot rescue the impaired cell growth or mitochondrial respiration ${ }^{16,43-45}$, indicating that mitochondrial GLS-catalyzed cleavage of the gammanitrogen of glutamine is essential for glutaminolysis. A possible reason for this requirement is the charge difference between glutamine and glutamate. Glutamine is a neutral amino acid and thus does not induce a negative charge burden in the mitochondrial matrix, which is already more negatively charged than the cytosol. Glutamate, however, is a negatively charged amino acid, and most cancer cells export-instead of import-glutamate ${ }^{46}$. Glutamate efflux is more crucial when NRF2 is activated. In cells with NRF2 activation, most glutamate is secreted, and cystine is imported by the SLC7A11 (xCT) antiporter mechanism $^{47}$ (Fig. 3).

Glutamate is also utilized to synthesize the antioxidant glutathione ${ }^{4}$. The first reaction in glutathione synthesis is the ligation of glutamate and cysteine catalyzed by glutamate-cysteine ligase (GCL). Next, glycine is added by glutathione synthetase (GSS). Additionally, glutamate can be converted to glycine through a transamination reaction catalyzed by phosphoserine aminotransferase (PSAT1) 
into phosphoserine (3-PS) and $\alpha$-KG. Phosphoserine is subsequently converted to glycine via serine hydroxymethyltransferase (SHMT) (Fig. 3). In cancer cells, the use of glutamate-derived nitrogen for NEAA production may be favored in various types of cancer cells to preserve nitrogen for anabolic reactions ${ }^{48}$ and may prevent apoptosis induced by ATF4 activation upon glutamine deprivation ${ }^{6}$.

\section{Asparagine}

Asparagine can be synthesized de novo from glutamine via asparagine synthetase (ASNS). Interestingly, asparagine was reported to be able to rescue cancer cells from glutamine deprivation-induced apoptosis ${ }^{43}$. This finding is surprising because asparagine supplementation does not restore the levels of other NEAAs (alanine, proline, and glutamate) or any TCA cycle intermediates ( $\alpha$-KG, malate, and fumarate). Instead, asparagine supplementation enhances the expression of glutamine synthetase (GLUL) and increases intracellular glutamine usage via glutaminolysis, resulting in the recovery of global protein translation that is blocked by glutamine deprivation ${ }^{45}$. These studies suggest that most glutamine-dependent protein translation activities can still proceed under asparagine supplementation in a glutamine-deprived environment, although the exact mechanism is still unknown. Furthermore, studies performed in endothelial cells, Kaposi's sarcoma-associated herpesvirus (KSHV)transformed cancer cells and several normal fibroblast or epithelial cell lines reported a similar effect of asparagine on supporting cell survival and protein translation after glutamine deprivation ${ }^{44,49,50}$. Interestingly, high intracellular asparagine levels have recently been identified to be essential for breast cancer metastasis ${ }^{51}$. This study suggested that L-asparaginase treatment alone can reduce the incidence of breast cancer metastasis to the lung without affecting primary tumor growth. Although the clinical effect of L-asparaginase clearly indicates that asparagine is crucial for tumor survival and metastasis ${ }^{52}$, the importance of asparagine beyond protein synthesis and the mechanism by which asparagine supplementation enhances glutamine-associated metabolism are less well understood. Recently, asparagine has been reported to function as an exchange factor needed for the uptake of other amino acids that are required for mTORC1 activation $^{53}$ and for enhanced nucleotide biosynthesis under mitochondrial electron chain transport system impairment ${ }^{54}$. Further investigation is needed to explain the considerable mechanistic importance of asparagine in cancer metabolism.

\section{Redox control of glutamine}

A low level of reactive oxygen species (ROS) activates tumorigenic growth signaling; however, when the level exceeds the cellular redox capacity, ROS can damage macromolecules such as proteins, lipids and nucleotides $^{55}$. Recent studies suggest that cancer cells are under increased oxidative stress caused by oncogenic transformation, leading to metabolic alterations that result in ROS production ${ }^{56}$. Under these conditions, glutamine metabolism becomes essential for maintaining cellular redox homeostasis by harnessing enhanced ROS levels. The metabolic pathway by which glutamine mitigates ROS is the glutathione synthesis pathway ${ }^{57}$ (Fig. 3). Glutathione is a tripeptide (Glu-Cys-Gly) that deactivates peroxidefree radicals. Glutamine is considered the rate-limiting factor in glutathione synthesis ${ }^{58,59}$. Indeed, experiments using uniformly labeled ${ }^{13} \mathrm{C}$-glutamine showed that glutathione was enriched with five ${ }^{13} \mathrm{C}$ atoms in glutathione, suggesting that glutamine is the major source of glutathione $\mathrm{e}^{16,57,60}$. As shown in Fig. 3, glutamine is a direct fuel for the use of glutathione as a source of glutamate and is indirectly responsible for cystine uptake via the $\mathrm{xCT}$ antiporter system, which takes up cystine and simultaneously secretes glutamate ${ }^{61}$. Consistent with this observation, glutamine starvation has been associated with impaired uptake of cystine through $\mathrm{xCT}$ and decreased intracellular glutathione levels ${ }^{62}$. Furthermore, cells in several types of cancers are characterized by significant enhancement of glutathione biosynthesis, and this metabolic vulnerability has been targeted to sensitize these cancer cells to ROS-induced drugs ${ }^{63}$.

Glutathione can be recovered from its oxidized form, accompanied by the conversion of NADPH to $\mathrm{NADP}^{+}$. In pancreatic cancer cells, glutamine supports the production of NADPH via a noncanonical metabolic pathway ${ }^{29}$, and the mitochondrial glutamine transporter is strongly associated with glutaminolysis-induced NADPH generation ${ }^{16}$. In addition, IDH1-dependent reductive glutamine metabolism produces NADPH, which decreases mitochondrial ROS during anchorage-independent growth ${ }^{64}$. In summary, glutamine maintains cellular redox homeostasis by supplying fuels for glutathione synthesis and endowing reducing power in the form of NADPH for sustaining tumor growth.

\section{Control of glutamine metabolism by hypoxia}

Hypoxic conditions promote the uptake of glutamine by increasing the levels of glutamine transporters such as SLC1A5, the SLC1A5 variant, and SLC38A2 ${ }^{16,65}$ and switch the fate of glutamine from the oxidative pathway into the reductive carboxylation pathway ${ }^{24}$. This metabolic adaptation is critical because of the reduced entry of pyruvate into the TCA cycle by activated PDK1 and the increased lactate secretion in hypoxia ${ }^{66}$. Via this metabolic adaptation, cells can continually generate TCA metabolites, such as $\alpha-K G$ and citrate, which are converted to cytosolic acetyl-CoA for lipid biosynthesis (Fig. 4). 


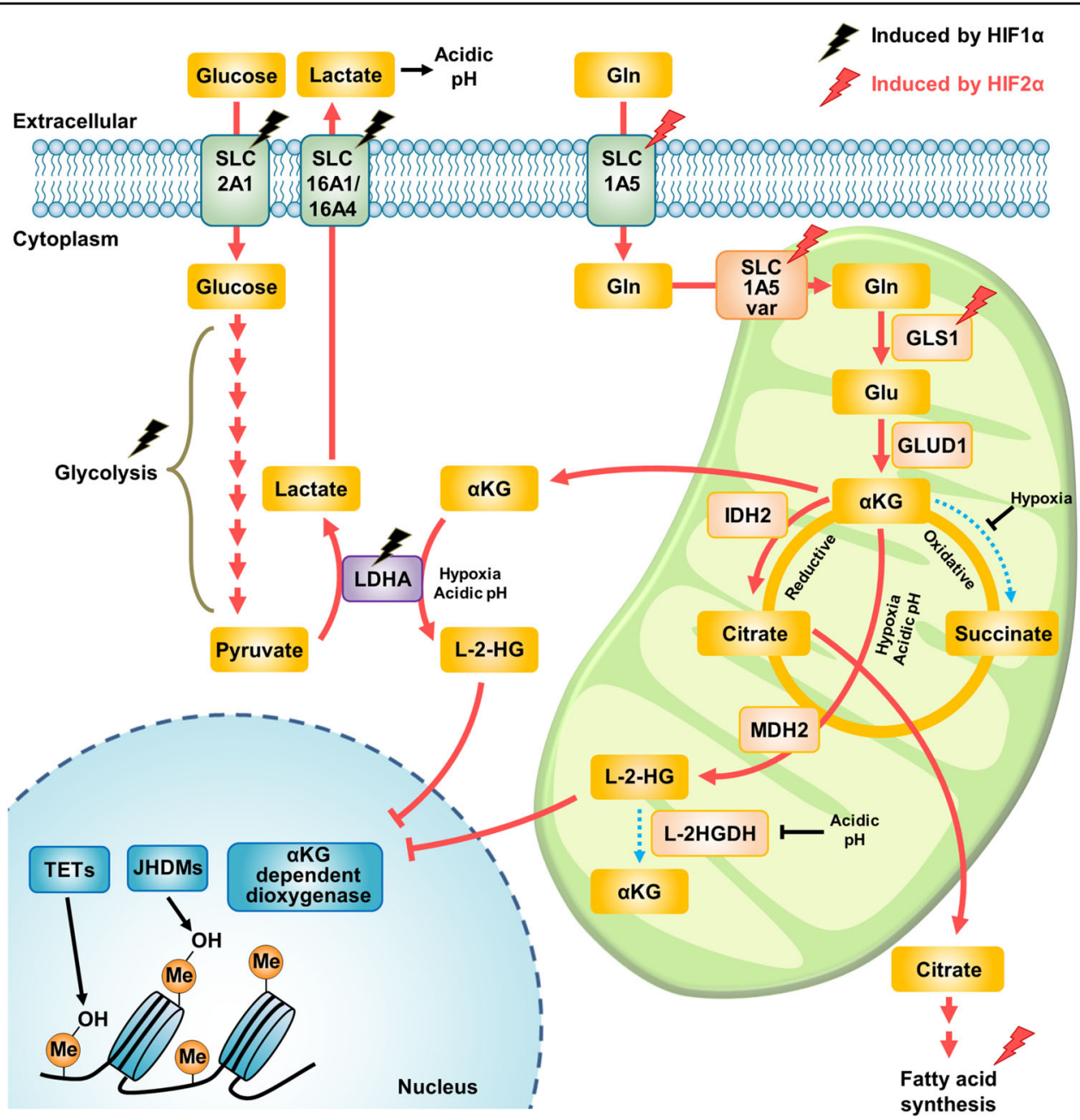

Fig. 4 Control of glutamine metabolism by hypoxia. Hypoxia stabilizes HIF-a proteins such as HIF-1a and HIF-2a. HIF-1a enhances glucose uptake and increases the level of glycolytic enzymes. Under hypoxic conditions, most glucose-derived pyruvate is converted into lactate via LDHA and exported to the extracellular space through the lactate transporters SLC16A1 and SLC16A4. Under these conditions, HIF-2a-mediated glutaminolysis becomes essential to support the adaptation to hypoxia, altering the metabolic fate of glutamine via reductive carboxylation to generate citrate. Then, citrate participates in fatty acid synthesis in the cytosol, which is also activated by stabilized HIF-2a. Hypoxia-induced acidic pH also plays a crucial role in the production of L-2-HG by affecting the substrate affinities of LDHA and MDH. Next, L-2-HG can control DNA or histone methylation levels by regulating a-KG-dependent dioxygenases. HIF hypoxia-inducible factor, GLS glutaminase, GLUD glutamate dehydrogenase, IDH isocitrate dehydrogenase, MDH malate dehydrogenase, L-2HGDH L-2-hydroxyglutarate dehydrogenase, LDHA lactate dehydrogenase, TETs ten-eleven translocation enzymes, JHDMs JmjC domain-containing histone demethylases, Gln glutamine, Glu glutamate, a-KG a-ketoglutarate, L-2-HG L-2hydroxyglutarate, Me methylation.

HIF- $\alpha$ is the most well-known transcription factor activated in hypoxia. HIF- $1 \alpha$ is activated due to blockade of its degradation pathway mediated by low oxygen levels, thereby increasing the expression of target genes, including those encoding glycolytic enzymes and glucose transporters, and increasing lactate secretion ${ }^{67}$. Although HIF- $2 \alpha$ has biochemical characteristics similar to those of HIF- $1 \alpha$, the metabolic role of HIF- $2 \alpha$ in a low-oxygen environment is relatively unknown ${ }^{68}$. Recently, hypoxiainduced expression of the SLC1A5 variant was shown to be mediated by HIF- $2 \alpha$ and to lead to metabolic reprogramming toward glutamine metabolism in pancreatic cancer cells ${ }^{16}$. Given that HIF- $2 \alpha$ is an important transcription factor in cancer progression and leads to poor prognosis $^{69,70}$, these findings suggest that targeting HIF$2 \alpha$ might be an effective therapeutic strategy by inhibiting glutamine metabolism in these notorious cancers. Furthermore, long-term exposure of cancer cells to acidic extracellular conditions induces metabolic reprogramming toward glutamine metabolism via HIF- $2 \alpha$ activity $^{71}$. In addition, extracellular lactate stabilizes HIF-2 $\alpha$, and HIF- $2 \alpha$ then transactivates MYC, increasing the levels of glutamine transporters and GLS1, in turn resulting in increased glutamine catabolism ${ }^{72}$. These findings indicate 
that just as HIF- $1 \alpha$ generally affects glucose metabolism in hypoxia, HIF- $2 \alpha$ also plays a distinct role in glutamine metabolism to promote metabolic adaptation in hypoxia (Fig. 4).

Fatty acid synthesis is an anabolic process that uses cytosolic citrate to produce acetyl- $\mathrm{CoA}^{73}$. Glutamine acts as an alternative fuel for fatty acid synthesis, supplying citrate via mitochondrial reductive carboxylation, especially under hypoxic conditions ${ }^{74,75}$. In the context of constitutive HIF- $2 \alpha$ stabilization $^{75}$ or a defective mitochondrial electron transport chain ${ }^{76}$, glutamine-derived $\alpha-K G$ is reductively carboxylated through the consumption of NADPH by IDH2 to generate citrate. Next, mitochondrial citrate is transported across the inner mitochondrial membrane via a citrate carrier (CIC or SLC25A1) to support fatty acid synthesis for tumor progression in hypoxia ${ }^{73}$ (Fig. 4). This mechanism is very important in clear cell renal cell carcinoma (ccRCC) in which HIF- $2 \alpha$ signaling is constitutively activated and intracellular lipid droplets are abundant. Fatty acid synthesis induced by HIF- $2 \alpha$ is crucial for cell viability in ccRCC by sustaining endoplasmic reticulum (ER) homeostasis $^{77}$. Furthermore, HIF-2 $\alpha$ represses the transcription of carnitine palmitoyltransferase $1 \mathrm{~A}$ (CPT1A), which is responsible for mitochondrial $\beta$-oxidation by transporting fatty acids and results in lipid deposition ${ }^{78}$. Indeed, recent studies have shown that HIF- $2 \alpha$ can be targeted by selective inhibitors and have indicated that these molecules effectively suppress cancer cell growth and tumor angiogenesis characteristics in ccRCC ${ }^{79-82}$. Thus, HIF-2 $\alpha-$ induced fatty acid synthesis using glutamine-derived citrate can be therapeutically targeted in several cancers, especially ccRCC.

In several cancers, glutamine metabolism is closely related to hypoxia-induced chemoresistance ${ }^{83}$. For example, glutamine depletion has been shown to abolish hypoxia-induced chemoresistance in cholangiocarcinoma. Impairing glutamine metabolism also induces sensitivity in gemcitabine-resistant pancreatic cancer cells ${ }^{16,84,85}$. This bolstered chemoresistance in cancer cells is partially supported by glutathione synthesis via glutaminolysis ${ }^{86}$. Given the importance of glucose and glutamine metabolism in pancreatic cancer cells, it is not surprising that gemcitabine resistance is closely associated with metabolic status, including cellular glucose and glutamine levels. Hypoxia increases the deoxycytidine triphosphate (dCTP) level through the pentose phosphate pathway (PPP) via glucose metabolism and results in resistance to gemcitabine, a dCTP analog ${ }^{87}$. Furthermore, redox modulation augmented by increased glutathione synthesis from glutamine was reported to be the mechanism of resistance to gemcitabine in pancreatic cancer cells ${ }^{16}$. Consistent with these findings, while NRF2 induces chemoresistance in KRAS-driven cancers, suppressing glutamine metabolism leads to weakened chemoresistance in these cancer cells ${ }^{85}$. These studies suggest that targeting glutamine metabolism can be an effective cancer treatment strategy when combined with conventional anticancer chemotherapy.

Under hypoxic conditions, L-2-hydroxyglutarate (L-2$\mathrm{HG}$ ) was proven to be generated by lactate dehydrogenase A (LDHA) and malate dehydrogenase $(\mathrm{MDH})^{88,89}$. Under normal physiological conditions, LDHA catalyzes the conversion of pyruvate to lactate. However, under hypoxic conditions, LDHA can produce L-2-HG. The cellular metabolic alteration of increased L-2-HG levels contributes to the regulation of histone and DNA methylation levels by inhibiting epigenetic modification enzymes that use $\alpha$-ketoacid as a cofactor. These events mitigate cellular reductive stress by suppressing key metabolic pathways, indicating a crucial role of L-2-HG. Acidic $\mathrm{pH}$ has also been reported to induce L-2-HG production via the promiscuous activity of LDHA and MDH enzymes. Acidic $\mathrm{pH}$ impairs the activity of the mitochondrial L-2-HG removal enzyme L-2-hydroxyglutarate dehydrogenase (L2HGDH) and enhances the protein stabilization of HIF$1 \alpha$, leading to its escape from the degradation pathway ${ }^{90}$. In addition, L-2-HG accumulation in an acidic $\mathrm{pH}$ environment has been reported to result in HIF- $1 \alpha$ stabilization in normoxia ${ }^{91}$ (Fig. 4).

Homozygous L2HGDH mutations in germline transmission cause a disease named 2-hydroxyglutaric aciduria $(\mathrm{L}-2-\mathrm{HGA})^{92}$. L-2-HGA is an autosomal recessive encephalopathy with an onset in childhood that causes developmental delays, epilepsy and cerebellar ataxia, the traditional clinical signs of this condition. Interestingly, patients with L-2-HGA are affected by tumors, including brain tumors ${ }^{93}$, bone tumors ${ }^{94}$, and nephroblastoma (Wilms tumor) $^{95}$. Furthermore, increased L-2-HG levels caused by reduced expression of L2HGDH have been reported in renal cancer ${ }^{96}$. These studies indicate an oncogenic effect of L-2-HG and the association of L-2HG with tumorigenesis under hypoxic conditions.

\section{Control of epigenetic changes by glutamine}

The metabolic state constitutes a fundamental component of chromatin modification and genome regulation ${ }^{97}$. As metabolites are the substrates used to generate chromatin modifications, including methylation and acetylation modifications of histones, a complicated interaction exists between metabolism and epigenetics. In particular, glutamine-derived $\alpha$-KG has been implicated in regulating cellular histone and DNA methylation levels ${ }^{98}$.

$\alpha-K G$, also named 2-oxoglutarate, is a cofactor for 2oxoglutarate-dependent dioxygenases (2-OGDDs), which catalyze hydroxylation reactions on diverse substrates. The activities of 2-OGDDs are affected by the intracellular level of $\alpha-K G$, succinate, fumarate, or 2-HG. These 


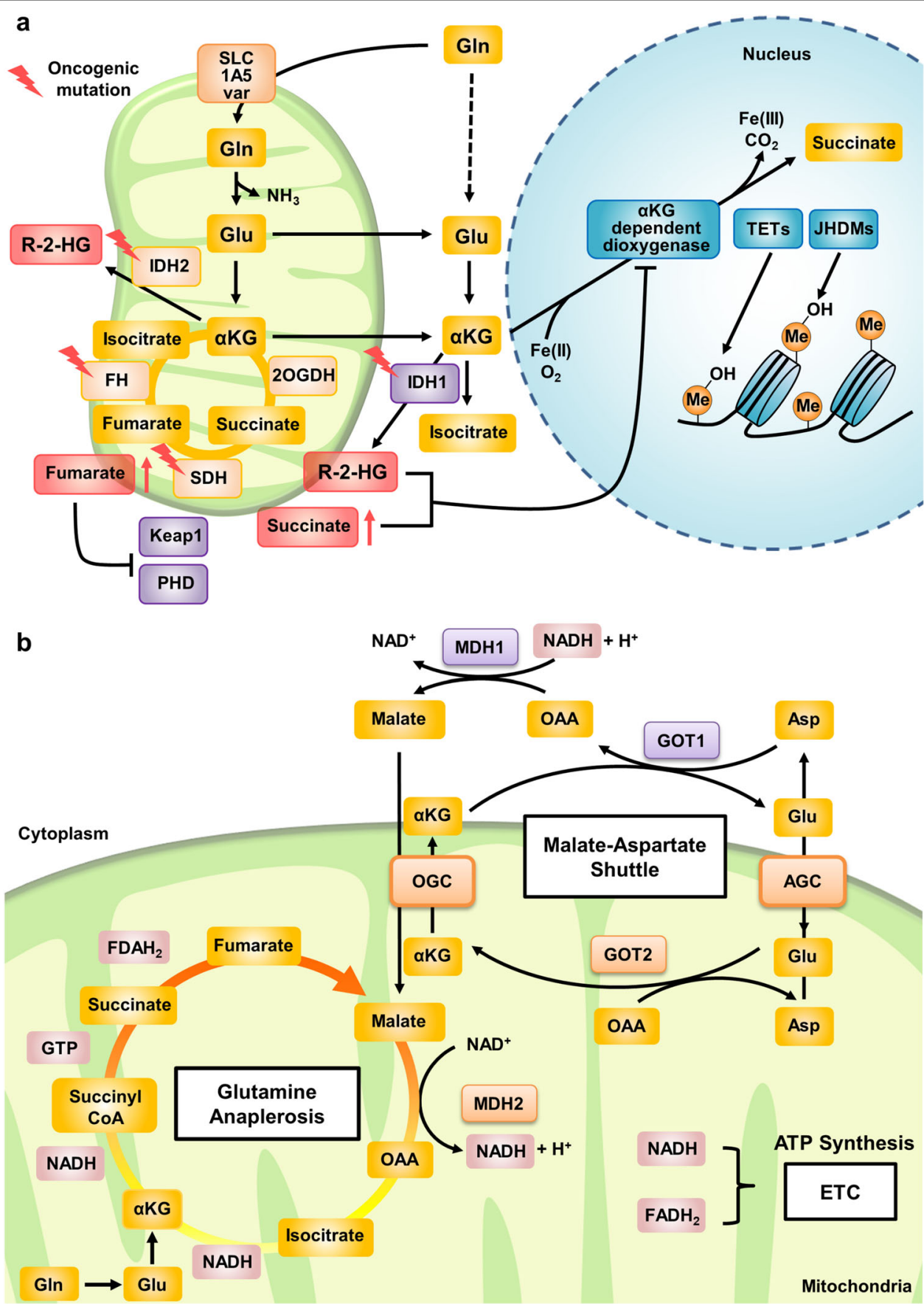

Fig. 5 (See legend on next page.)

hydroxylation reactions also require $\mathrm{Fe}^{2+}$ as a cofactor, $\mathrm{O}_{2}$ as a cosubstrate and ascorbic acid (vitamin $\mathrm{C}$ ) as a reductase, which restore the activity of 2-OGDD enzymes (Fig. 5a). Among 2-OGDDs, Jumonji C domaincontaining histone demethylases and ten-eleven translocation (TET) family DNA demethylases are major enzymes that induce epigenetic modifications using glutamine-derived $\alpha-K G$. In these reactions, $\alpha-K G$ is oxidized to succinate, and increasing levels of succinate can suppress the progression of $\alpha$-KG-dependent histone or DNA demethylase reactions ${ }^{98}$.

In cancer cells, mutations in succinate dehydrogenase subunit B (SDHB) cause susceptibility to familial pheochromocytoma ${ }^{99}$ and familial paraganglioma ${ }^{100}$ as well as 
(see figure on previous page)

Fig. 5 Glutamine oncometabolites and energy production from glutamine. a Several mutations in enzymes in the glutaminolysis pathway are responsible for the production of oncometabolites. Mutation of IDH1 and IDH2 produces R-2-HG from a-KG, which, when accumulated, leads to the inhibition of dioxygenases, in turn leading to the activation of TET and JHDM enzymes inside the nucleus. Mutation of SDH arrests the TCA cycle, resulting in an increase in the succinate concentration. A high concentration of succinate has an effect similar to the oncometabolite effect of R-2-HG. Additionally, impaired function of $\mathrm{FH}$ prevents further metabolism of fumarate, leading to its accumulation. FH impairment inhibits the function of Keap1 and PHD, which stimulates the transcription of protooncogenes. Gln glutamine, Glu glutamate, a-KG a-ketoglutarate, IDH isocitrate dehydrogenase, 2OGDH 2-oxoglutarate dehydrogenase, SDH succinate dehydrogenase, FH fumarate hydratase, R-2-HG R-2-hydroxyglutarate, Keap1 Kelch-like ECH-associated protein 1, PHD prolyl hydroxylase, TETs ten-eleven translocation enzymes, JHDMs JmjC domain-containing histone demethylases, Me methylation. b. Glutamine anaplerosis is a key mitochondrial metabolic pathway for cancer cell growth and survival. Influx of glutamine-derived a-KG into the TCA cycle replenishes the intermediates and consequently generates NADH, FADH 2 , and GTP. The generated GTP can be readily converted to an equal amount of ATP. Additionally, glutamate and a-KG produced via glutaminolysis participate in the malateaspartate shuttle, promoting the transport of $\mathrm{NADH}$ from the cytosol into mitochondria. Elevated mitochondrial $\mathrm{NADH}_{\text {and }} \mathrm{FADH} \mathrm{H}_{2}$ levels collectively contribute to enhanced ATP production via OXPHOS through the ETC. Gln glutamine, Glu glutamate, Asp aspartate, aKG a-ketoglutarate, GOT1/2 glutamic-oxaloacetic transaminase 1/2, MDH1/2 malate dehydrogenase 1/2, OAA oxaloacetate, OGC 2-oxoglutarate carrier, AGC aspartate-glutamate carrier, ETC electron transport chain.

gastrointestinal stromal tumors ${ }^{101}$. An increased ratio of succinate to $\alpha-K G$ in cancers resulting from impaired succinate dehydrogenase $(\mathrm{SDH})$ activity is related to pervasive DNA hypermethylation, which contributes to the downregulation of key genes implicated in cell differentiation and cancer stages ${ }^{102}$. Moreover, the core region of solid tumors exhibits a deficiency of glutamine compared with other amino acids. This severe glutamine deprivation leads to dramatic histone hypermethylation due to decreased $\alpha-K G$ levels subsequent to decreased activity of Jumonji domain-containing histone demethylases and results in cancer cell dedifferentiation and resistance to BRAF inhibitors ${ }^{103}$.

In addition to its role in cancer cells, $\alpha-K G$ supports the self-renewal of naive murine embryonic stem cells (mESCs) by promoting histone and DNA demethylation $^{104}$. In addition, at later stages of pluripotency, $\alpha-K G$ derived from glutamine can promote early differentiation, suggesting that the stage of cellular maturity can alter the effect of $\alpha-K^{105}$. Furthermore, PSAT1 regulates changes in the level of glutamine-derived $\alpha-K G$, which controls mESC pluripotency and differentiation ${ }^{106}$. These reports suggest that $\alpha-K G$ generated via glutaminolysis is closely related to the cellular decisions that characterize stem cells. In skeletal stem cells (SSCs), GLS and glutamine metabolism are required for the regulation of osteoblast and adipocyte specification and physiological bone formation $^{107}$. In macrophage cells, $\alpha-K G$ produced via glutaminolysis promotes M2 activation via Jmjd3-dependent metabolic and epigenetic reprogramming ${ }^{108}$.

In $\mathrm{T}$ cell activation, glutamine deprivation has been shown to alter the activation of naive $\mathrm{CD}^{+} \mathrm{T}$ cells and result in their differentiation into forkhead box P3-positive $\left(\right.$ Foxp $\left.3^{+}\right)$regulatory $\mathrm{T}\left(\mathrm{T}_{\text {reg }}\right)$ cells, which have suppressor functions ${ }^{109}$. Recently, glutamine metabolism has been shown to be linked to white adipose tissue (WAT) inflammation in obesity ${ }^{110}$. The researchers discovered that glutamine metabolism is impaired in the obese state, leading to increased chromatin O-GlcNAcylation and activation of genes in proinflammatory pathways.

Collectively, glutamine-derived metabolites act as epigenetic modulators in a wide range of cell and tissue types, including various types of cancer cells, stem cells, immune cells, and even adipocytes. Considering that the SLC1A5 variant is an important regulator of the production of glutamine-derived $\alpha-\mathrm{KG}^{16}$, confirming whether epigenetic regulation by glutamine-derived $\alpha-K G$ is affected by the SLC1A5 variant in cancer cells or stem cells is necessary (Fig. 5a).

\section{Glutamine and its oncometabolites}

The discovery of R-2-hydroxyglutarate (R-2-HG) accumulation in several tumors encouraged investigators to initially establish the term "oncometabolite"111. Genetic and metabolic studies have further shown that metabolites such as succinate and fumarate, which are generated under normal physiological conditions, are associated with tumorigenesis in several cancer types. Interestingly, these metabolites were often found to be associated with glutamine metabolism ${ }^{112}$. In particular, the production of these oncometabolites was affected by the level of glutamine-derived $\alpha-K G$. Although additional studies are needed, ample experimental data support the recognition of R-2-HG, succinate, and fumarate as oncometabolites.

\section{R-2-HG}

Wild-type IDH1 and IDH2 catalyze the reaction by converting isocitrate and $\mathrm{NADP}^{+}$into $\alpha-\mathrm{KG}$ and $\mathrm{CO}_{2}$ with the concomitant generation of $\mathrm{NADPH}$ in the cytosol and mitochondrial matrix. However, mutant IDH enzymes convert $\alpha-K G$ into $\mathrm{R}-2-\mathrm{HG}$ with the oxidation of $\mathrm{NADPH}$ into $\mathrm{NADP}^{+}$. Thus, various tumors, including glioma, secondary glioblastoma, and acute myeloid leukemia (AML), harboring heterozygous point mutations in the active sites of IDH1/2 show dramatic increases in the $\mathrm{R}-2-\mathrm{HG}$ levels ${ }^{111,113-115}$. A high level of $\mathrm{R}-2-\mathrm{HG}$ is 
sufficient to cause leukemia to arise from hematopoietic cells by maintaining their dedifferentiation and proliferation activities ${ }^{116}$. The role of R-2-HG as an oncometabolite has been implicated in epigenetic modifications through the inhibition of $\alpha$-KG-dependent dioxygenases and demethylases, which has been assumed to be a driver of tumorigenesis ${ }^{117,118}$. In addition, dysregulated $\alpha-K G$ flux from normal reductive anabolism via the TCA cycle toward R-2-HG production has been associated with other metabolic flux impairments and disrupted redox balance ${ }^{119,120}$ (Fig. 5a).

Interestingly, the generation of R-2-HG from glutamine has been proven to occur rapidly in patient-derived chondrosarcoma cell lines harboring endogenous IDH mutations, indicating fundamental metabolic differences between cells that harbor IDH1/2 mutations and those that do not ${ }^{121}$. In this study, glutamine flux was directed toward the generation of R-2-HG in IDH1/2 mutant cells, and the kinetics of R-2-HG formation were proportionally of the same order of magnitude as those of glutamate or $\alpha-K G$ formation via glutaminolysis. Indeed, glutaminederived R-2-HG accumulates and prevents the differentiation of myeloblasts, resulting in uncontrolled growth of blood cells ${ }^{122}$. After FDA approval of enasidenib, a first-in-class drug targeting cancer metabolism via inhibition of IDH2 activity, more studies were conducted with R-2-HG positioned as an oncometabolite. CB-839, a GLS inhibitor that blocks the conversion of glutamine into glutamate, reduced the production of $\mathrm{R}-2-\mathrm{HG}$ in $\mathrm{AML}$ cell lines and patient tissues harboring IDH1/2 mutations ${ }^{123}$. As the importance of R-2-HG in boosting tumor initiation, proliferation and metastasis is emphasized, identifying whether metabolic enzymes or transporters associated with glutamine metabolism could be involved in the generation of R-2-HG is interesting.

\section{Succinate}

The normally functioning SDH enzyme is localized in the inner mitochondrial membrane and plays a role in the electron transport chain as well as the conversion of succinate into fumarate. In 2008, mutation of SDH was discovered in cancers such as paraganglioma and pheochromocytoma cells ${ }^{124}$. Later, similar observations were made in gastrointestinal tumors, neuroblastomas, renal tumors, thyroid tumors, and testicular tumors ${ }^{125,126}$. Several research groups have focused on the mechanism that underlying the features of tumorigenesis and cancer cell survival in the setting of SDH mutations. As succinate accumulates via the inhibition of the 2-OGDD enzyme, epigenetic modification acts in the process of cell transformation into a hypermethylated phenotype ${ }^{100}$. Several studies have shown that SDH-deficient cells exhibit increased tumorigenesis and that this increase is reversed by the addition of $\alpha-K G$, supporting the idea that succinate accumulation contributes to tumorigenesis through epigenetic modification ${ }^{100}$. Succinate-specific effects are initiated by epigenetic alterations through the inhibition of KDMs and the TET family $5 \mathrm{mC}$ hydroxylases, which induce the translation of tumorigenic genes (Fig. 5a). The other mechanism by which succinate supports tumorigenesis acts through the inhibition of hypoxia-inducible factor prolyl hydroxylase (PHD). PHD activates the pseudohypoxic response by stabilizing HIF$1 \alpha$, which is a well-known tumorigenesis enhancer, and as a transcription factor, maintains the metabolic reprogramming of cancer cells to support their survival ${ }^{127}$. In addition to the tumorigenic effects of succinate accumulation, SDH5 mutation is the key driver supporting the acquisition of epithelial-mesenchymal transition (EMT) characteristics. The results of a clinical study further confirmed this observation by showing that patients with nonmetastatic lung cancer harbored loss-of-function mutations in $\mathrm{SDH} 5^{128}$. The study of succinate as an oncometabolite has only recently begun, and more research needs to be conducted to completely understand its tumorigenic properties.

\section{Fumarate}

Fumarate is another example of an oncometabolite produced by the action of fumarate hydratase on succinate. In 2001, mutation of fumarate hydratase leading to its inactivation was discovered in renal cell cancer ${ }^{129}$. Mutation of this enzyme leads to fumarate accumulation not only in skin cancer and uterine leiomyomas but also in breast, bladder, and Leydig cell tumors ${ }^{130}$. Further confirmation of fumarate as an oncometabolite was verified by experimental data showing that tumor cells lost their ability to invade and migrate when the function of fumarate hydratase was restored by an external expression vector $^{131}$. In attempts to understand the cause of these effects, it was found that cells with high concentrations of fumarate display a phenotype of DNA hypermethylation. In addition, fumarate inhibits TET enzymes, which stimulate EMT, leading to cancer metastasis ${ }^{131,132}$. Similar to succinate, fumarate contributes to the inactivation of PHD, stabilizing HIF proteins to promote cell survival ${ }^{133}$ (Fig. 5a). In addition, accumulated fumarate can participate in different reactions of the addition of a succinate group to the thiol group of various proteins. For example, in hereditary leiomyomatosis and renal cell cancer (HLRCC), a high level of fumarate caused by genetic mutation of fumarate hydratase induces the succination of Kelch-like ECH-associated protein 1 (KEAP1) accompanied by the consumption of a fumarate molecule ${ }^{134,135}$. Endogenously, succinylated KEAP1 dissociates from the NRF2 protein to help cancer cells survive stress. High concentrations of fumarate bind to glutathione, augmenting ROS signaling and accumulation, as observed in 
not only in vitro models but also in vivo models ${ }^{136,137}$. Additionally, high levels of fumarate react with the cysteine group of mitochondrial aconitase-2 and ironsulfur cluster binding protein-2, facilitating cellular metabolic adaptation to stresses ${ }^{138}$. The importance of fumarate hydratase mutation for cancer survival and growth is being studied in depth to completely understand the role of fumarate as a tumorigenic oncometabolite. This knowledge will aid in the complete comprehension of cancer metabolism.

\section{Glutamine-derived energy production}

The influx of $\alpha-K G$ into the TCA cycle and its subsequent oxidization generates two molecules of NADH and one molecule of $\mathrm{FADH}_{2}$ from the series of reactions catalyzed by $\mathrm{OGDH}, \mathrm{SDH}$, and MDH. Additionally, when succinyl-CoA is converted to succinate by succinate thiokinase, one molecule of GTP is generated, which can be readily converted to ATP by nucleoside-diphosphate kinase (NDPK). NADH and FADH2 produced via glutaminolysis are then fed into the electron transport chain to create the electrochemical gradient necessary for ATP production via oxidative phosphorylation ${ }^{139,140}$ (Fig. 5b). Correspondingly, in K-Ras mutant cells, the oxygen consumption rate and ATP generation are enhanced by glutamine, contributing to tumorigenesis ${ }^{55}$. Moreover, after the activation of K-Ras and Akt in transformed cells, $60 \%$ of the total $\mathrm{FADH}_{2}$ and $\mathrm{NADH}_{2}$ are synthesized from glutamine, while only $30 \%$ is derived from glucose ${ }^{140}$. Additionally, the level of the mitochondrial glutamine transporter controls the cellular ATP level stimulated by glutamine, suggesting that glutamine is an important energy source via mitochondrial glutaminolysis ${ }^{16}$. Collectively, these observations indicate that anaplerotic glutamine metabolism is highly responsible for energy generation in cancer cells.

Additionally, NADH can be generated by fatty acid oxidation (FAO) in the cytoplasm in tissues with high energy demand, such as cardiac muscle tissues, as well as in cancer cells ${ }^{141}$. Recent studies have suggested that in cancer cells with elevated cytosolic NADH levels, the malate-aspartate shuttle (MAS) actively takes up NADH to produce ATP in mitochondria through the electron transport chain ${ }^{142}$. The MAS comprises MDH1/2, GOT1/ 2 , the malate- $\alpha-K G$ antiporter and the glutamateaspartate antiporter, which exchanges mitochondrial $\alpha$ KG for cytosolic malate that is synthesized from oxaloacetic acid (OAA) by cytosolic MDH (Fig. 5b). Glutamate and $\alpha-K G$ serve as important exchangers in the MAS, and since GLS1 knockdown significantly suppresses NADH and ATP production in cancer cells ${ }^{143}$, the supply of glutamate and $\alpha-K G$ for the induction of MAS activity is evidently critical for ATP production in cancer cells (Fig. 5b).

\section{Glutamine metabolism upon cellular stresses}

Glutamine is the most abundant amino acid in the blood. During cellular stress, such as nutrient starvation and catabolic stress after trauma, surgery, infection, sepsis, or cancer cachexia, blood glutamine levels are severely decreased ${ }^{144}$. Under these conditions, several studies have reported that glutamine supplementation can offer a therapeutic approach for these critical illnesses ${ }^{145-147}$. Glutamine has been considered an immunomodulatory amino acid in several disease states, yet the mechanisms underlying the therapeutic effects of glutamine supplementation in critical illness remain poorly understood. Conceivably, glutamine could exert its beneficial effects by producing glutathione for redox homeostasis, maintaining nitrogen balance, or other functions in immune cells ${ }^{2}$.

Consistent with the importance of glutamine in stressful situations, glutamine deprivation induces cellular stress. Upon glutamine starvation, p53 activity is induced and can help cancer cells adapt to nutrient starvation through diverse mechanisms ${ }^{148}$. Recently, SLC1A3, as a crucial effector of p53, has been shown to support cell survival and growth in the absence of glutamine ${ }^{149}$. Under DNA damage such as radiation, glutamine is conditionally essential to support the synthesis of nucleotides and redox homeostasis. It has recently been demonstrated that radioresistant cancer cells reprogram metabolic flux toward glutamine anabolism. Under these conditions, cancer cells highly express glutamine synthetase, facilitating cancer cell growth under radiation stress ${ }^{150}$. Moreover, evidence has shown that during the DNA damage response, normal cells show a decrease in glutaminolysis controlled by SIRT4 protein suppressing GLUD1. In the absence of SIRT4, a failure to undergo cell cycle arrest induced by DNA damage causes a delay in DNA repair and increased chromosomal instability, suggesting a tumor suppressor effect of SIRT $4^{151}$.

Numerous studies have described the presence of alternative adaptive pathways upon the perturbation of glutamine metabolism. For instance, a recent study has shown that GLS1 inhibition induces an increase in mitochondrial glutamate-pyruvate transaminase 2 (GPT2) to assist in TCA cycle anaplerosis for sustaining cancer cell growth and survival ${ }^{152}$. Of note, GLS1 inhibition causes an elevation of the ROS level and induces GPT2 expression via ATF4, which again implies the importance of ATF4-mediated metabolic adaption during glutamine starvation.

Additionally, metabolic profiling has revealed that suppression of GLS1 induces a compensatory anaplerotic mechanism via pyruvate carboxylase (PC), which allows the release of a glutamine-independent supply of TCA intermediates by catalyzing the transformation of pyruvate to oxaloacetate $\mathrm{e}^{153}$. This PC-mediated alternative anaplerosis is considered important in specific types of 
cancers, including liver cancers and glioblastoma, for maintaining biosynthesis and redox homeostasis ${ }^{154-156}$. Collectively, cancer glutamine metabolism shows extraordinary flexibility and is intertwined with diverse metabolic pathways.

\section{Metabolic reprogramming induced by glutamine metabolism}

Unsurprisingly, glutamine metabolism plays a critical role in tumor progression since it not only supports mitochondrial oxidative phosphorylation but also supplies metabolic intermediates for the TCA cycle, glutathione synthesis, and NEAA synthesis and simultaneously produces NADPH ${ }^{157-159}$. Recently, glutamine was shown to be a major fuel for mitochondrial oxygen consumption in pancreatic cancer cells; in addition, the expression of the SLC1A5 variant affected the levels of metabolites derived from glucose metabolism, including lactate and ribulose5-phosphate, the intermediate metabolites in the $\mathrm{PPP}^{16}$. Intriguingly, this study regarding elevated glutamine metabolism in cancer cells also showed that glutaminolysis could in turn reinforce metabolic reprogramming, thus implying that glutamine metabolism plays a crucial role in tumorigenesis and tumor progression ${ }^{16}$ (Fig. 6a). Indeed, the process of adaptation to glutamine deprivation weakens the response to hypoxia, which normally strongly induces the expression of glycolytic enzymes ${ }^{83}$.

As previously described, glutamine is metabolized by mitochondrial enzymes into $\alpha-K G$, which serves as an important intermediate in the TCA cycle for anaplerosis. Furthermore, enhanced production of $\alpha-K G$ causes other critical effects, such as stimulation of the signaling pathways that support cell growth. $\alpha-K G$ induces mTORC1 activation by enhancing GTP loading of the RagB protein in a PHD-dependent manner, thus promoting cell growth $^{160,161}$. Accordingly, high mTORC1 activity in cancer cells promotes aerobic glycolysis and drives glucose addiction ${ }^{162,163}$ (Fig. 6b). In addition, mTORC1 activation via glutaminolysis suppresses autophagy and the DNA damage response ${ }^{164,165}$. Therefore, enhanced glutaminolysis might eventually contribute to the initiation and progression of cancer by stimulating cell growth via the mTORC1 pathway and enhancing aerobic glycolysis while disrupting the proper elimination of misfolded proteins, damaged DNA and organelles through the inhibition of autophagy and the DNA damage response ${ }^{166}$.

Enhanced glutaminolysis in cancer cells ensures a stable supply of glutamate and $\alpha-K G$ via sequential deamination processes inside mitochondria. Notably, ammonia is simultaneously generated as a byproduct of glutamine deamination. Hence, the facilitation of glutaminolysis leads to the accumulation of excess ammonia within cells, and a high concentration of ammonia is a potent inducer of autophagy ${ }^{167}$ (Fig. 6c). Although mTORC1 activation hinders autophagy, evidence has shown that autophagy can be upregulated in tumors with mTORC1 hyperactivation ${ }^{168}$. Therefore, glutaminolysis can suppress autophagy by activating the mTORC1 pathway but, on the other hand, can stimulate autophagy in the context of excess ammonia production. The fundamental need for ammonia-mediated induction of autophagy in cancer cells could be due to the cytoprotective functions of this event that allow cells to survive under extreme conditions ${ }^{166}$. Specifically, autophagy suppresses anoikis induced by the detachment of cancer cells from the extracellular matrix $(\mathrm{ECM})$ and hence promotes metastasis ${ }^{169}$. Furthermore, autophagy has been shown to promote glycolysis in hepatocellular carcinoma (HCC) cells by upregulating monocarboxylate transporter 1 (MCT1), which plays an important role in the transport of lactic acid ${ }^{170}$. Therefore, autophagy supports cancer progression and chemoresistance by allowing tumor cells to overcome both environmental and intracellular stress signals, including nutrient deprivation and chemotherapeutic cytotoxicities $^{167,171,172}$ (Fig. 6c).

However, the connection between glutamine and metabolic remodeling in cancer from the perspective of glucose metabolic flux, the mTORC1 pathway and autophagy has yet to be fully explored. This link might partially be explained by considering that the intimately entwined glucose and glutamine metabolic pathways cooperatively support the TCA cycle and that glutamine performs diverse functions for maintaining cellular homeostasis. Collectively, in-depth investigation of the role of glutaminolysis in tumor progression might hold the key for decoding cancer metabolic plasticity.

\section{Crosstalk between glutamine metabolism and oncogenic signaling}

The excessive proliferation exhibited by cancer cells demands a constant supply of fuels such as glucose and glutamine. Therefore, cancer cells orchestrate their metabolic pathways to coordinate their high demand for these nutrients. Metabolic reprogramming that promotes enhanced glutamine consumption in cancer cells is closely connected with dysregulation of oncogenes. Efforts have been undertaken to reveal the mechanism by which oncogenes modulate metabolic pathways that favor cancer cell growth ${ }^{173}$. Notably, cancer cells driven by oncogenic MYC, K-Ras, and PIK3CA require glutamine for their survival and display extensive anabolic utilization of glutamine ${ }^{29,174,175}$ (Fig. 7).

In cancer cells, genetic and epigenetic dysregulation of MYC expression and the loss of checkpoint components unleash the ability of MYC to promote cell growth, eventually leading to malignant transformation ${ }^{176}$. Oncogenic Myc stimulates mitochondrial glutaminolysis via transcriptional regulation of genes necessary for 


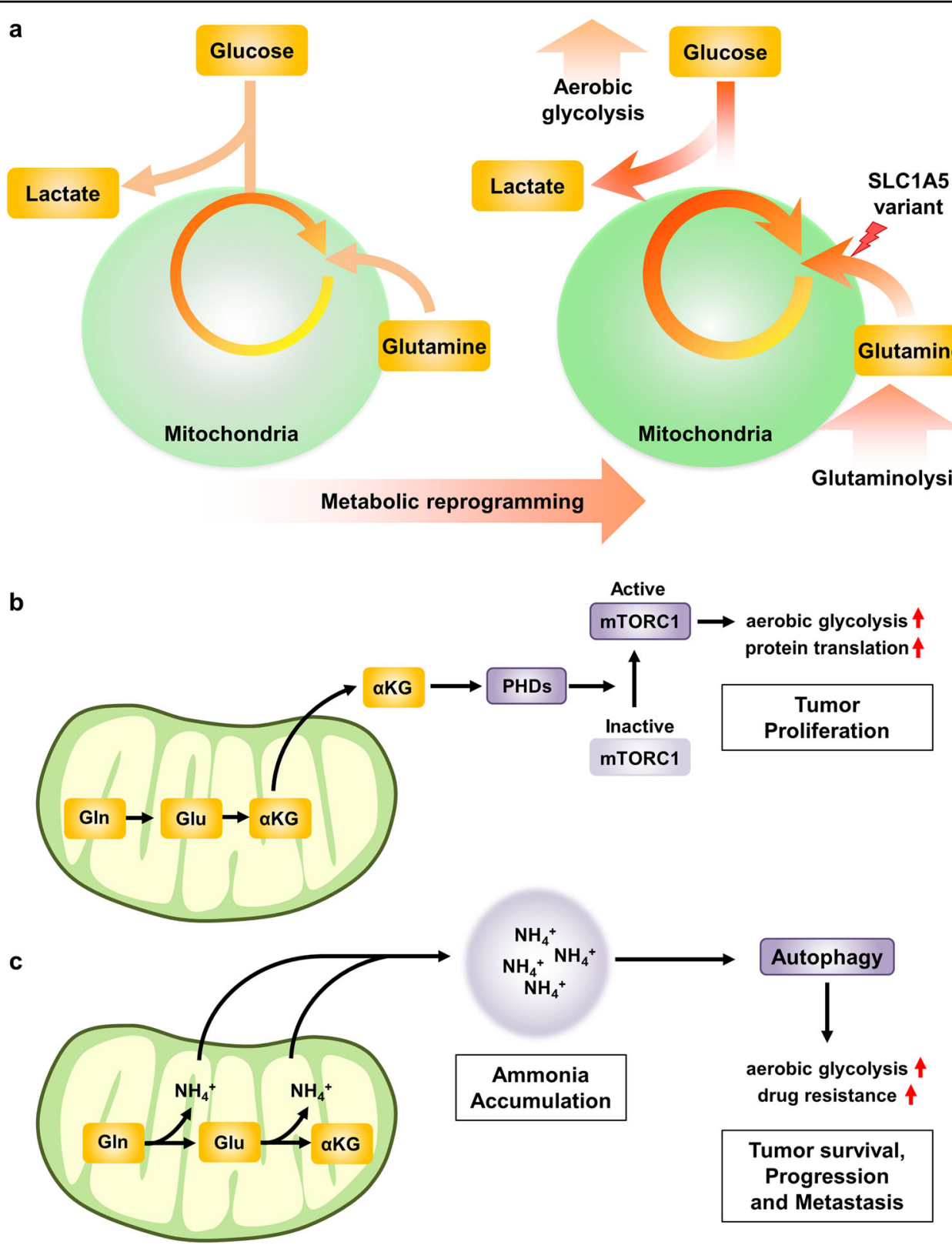

Fig. 6 Metabolic reprogramming induced by glutamine metabolism. a Aerobic glycolysis is a hallmark of cancer metabolism. During this process, most glucose-derived pyruvate is secreted extracellularly as lactate, and glutamine becomes a conditionally essential amino acid. Glutaminolysis sustains mitochondrial function, supplying TCA cycle metabolites such as aKG and generating diverse biomolecules, including NEAAs, $\mathrm{NADPH}$, and nucleotides. Increased glutamine flux into the mitochondrial matrix via the SLC1A5 variant can enhance glutaminolysis and lead to metabolic reprogramming toward enhanced aerobic glycolysis. b Glutamine-derived a-KG activates the mTORC1 signaling pathway, resulting in aerobic glycolysis and protein translation, which are crucial for tumor proliferation. c During glutaminolysis, ammonium ions are generated via a deamidation reaction catalyzed by glutaminase and glutamate dehydrogenase. Most ammonium ions are used as a nitrogen source for nucleotide biosynthesis and are disposed of via the urea cycle, but an excess of ammonium ions promotes autophagy. Augmented autophagy is associated with drug resistance by enhancing aerobic glycolysis and is involved in cancer cell survival, progression, and metastasis. Gln glutamine, Glu glutamate, aKG a-ketoglutarate, PHD prolyl hydroxylase.

cellular glutamine catabolism ${ }^{177}$. MYC-driven cancer cells exhibit enhanced glutamine utilization accompanied by increased expression of key glutaminolysis enzymes, including GLS1/GLS2 and GLUD1 ${ }^{178-180}$. Moreover,
MYC upregulates the glutamine transporter SLC1A5 to facilitate glutamine uptake into cells ${ }^{177}$. MYC-dependent enhancement of mitochondrial glutaminolysis leads to the reprogramming of mitochondrial metabolism to 


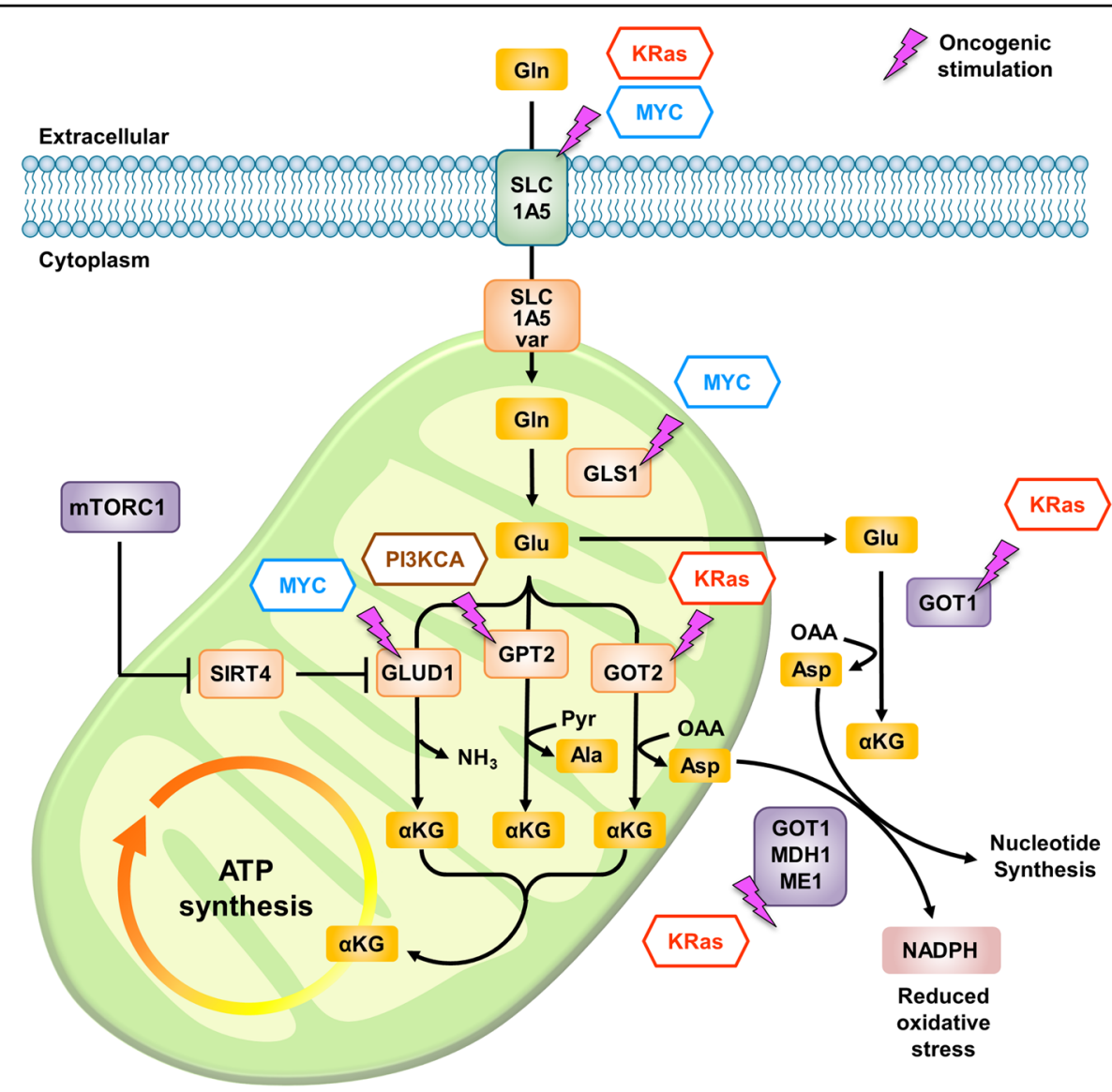

Fig. 7 Oncogenic control of glutamine metabolism. Oncogenes such as MYC, K-Ras, and PI3KCA modulate cancer metabolic reprogramming, favoring cancer cell growth and survival partially via the promotion of glutamine metabolism. Glutamine uptake is enhanced in MYC- and K-Rasdriven cells in which the expression of the glutamine transporter SLC1A5 is upregulated. Deamination of glutamine to form glutamate in mitochondria is enhanced by MYC-mediated upregulation of GLS1. Conversion of glutamate into a-KG is mediated by GLUD1 or aminotransferases such as GOT1/2 and GPT2. The expression of these enzymes is upregulated in cancer cells with MYC-driven, K-Ras-driven, and PI3KCA-driven signaling activation. Gln glutamine, Glu glutamate, Ala alanine, Asp aspartate, a-KG a-ketoglutarate, GLS1 glutaminase 1, GLUD1 glutamate dehydrogenase 1, GOT1/2 glutamic-oxaloacetic transaminase 1/2, GPT1/2 glutamic-pyruvate transaminase 1/2, MDH1 malate dehydrogenase 1, ME1 malic enzyme 1 .

accommodate the requirements for TCA cycle anaplerosis to sustain cellular viability and growth.

Similar to the situation in MYC-driven cancer cells, glutamine uptake is enhanced in K-Ras-driven cells via upregulation of SLC1A $5^{181}$. Additionally, K-Ras-driven cells are characterized by increased expression of GOT1 and GOT2 ${ }^{182,183}$. GOT1 and GOT2 catalyze the transamination reaction between oxaloacetate and glutamate to produce aspartate and $\alpha-K G$. Significantly, enhanced transamination and aspartate synthesis in K-Ras-driven cancer cells are important in the promotion of nucleotide biosynthesis ${ }^{184}$ and maintenance of redox balance ${ }^{29}$.

Intriguingly, the glutamine-dependent checkpoint at late G1 phase in the cell cycle is dysregulated in K-Rasdriven cancer cells ${ }^{185}$. In normal cells, the cell cycle is tightly regulated by various checkpoints. Nutrientdependent checkpoints regulate cell cycle passage through late G1 phase by sensing nutrient availability; glutamine is a particularly critical nutrient sensed in late G1 phase, and its deprivation causes cell cycle arrest at G1 phase $^{186}$. Importantly, activation of K-Ras in cancer cells results in bypass of the late G1 glutamine-dependent checkpoint. Specifically, glutamine deprivation in K-Rasdriven cancer cells leads to growth arrest in $\mathrm{S}$ or G2/M phase instead of in G1 phase. Consistent with this observation, K-Ras sensitizes cells to glutamine deprivation, and K-Ras knockdown rescues cells from apoptosis induced by low glutamine levels ${ }^{187}$. Collectively, these findings indicate that enhanced glutamine metabolism and cell growth dysregulation are established in K-Rasdriven cancer cells to promote uncontrolled cell growth and to assist with glutamine acquisition and utilization for cell growth.

The PI3K signaling pathway is dysregulated in many tumors, and analyses have shown that PIK3CA is an oncogene that also contributes to tumor progression 
partially via metabolic reprogramming ${ }^{188}$. Oncogenic PIK3CA increases the dependency of cancer cells on glutamine by upregulating the expression of mitochondrial GPT2, which catalyzes the transamination reaction that converts glutamate and pyruvate into $\alpha-K G$ and alanine $^{175}$. Thus, cells with PIK3CA mutations exhibit increased sensitivity to glutamine deprivation. Additionally, compared with wild-type cells, PIK3CA mutant colorectal cancer (CRC) cells exhibit elevated anaplerotic $\alpha$ KG production and ATP generation from glutamine.

In addition to oncogenic regulators, there are some key upstream regulators of glutamine metabolism that are widely recognized for their pivotal role during tumorigenesis. mTORC1, which is well known for its function at the center of cancer metabolic reprogramming, promotes mitochondrial glutaminolysis via the migration of SIRT4mediated inhibition of GLUD $1^{189}$. Specifically, mTORC1 promotes proteasome-mediated destabilization of cAMP response element binding-2 (CREB2) to suppress transcription of SIRT4. Accordingly, loss of SIRT4 enhances glutamine-dependent proliferation and genomic instability, which simultaneously contribute to tumorigenesis ${ }^{151}$.

Furthermore, mTORC1 also acts as a downstream effector of glutamine. Glutamine itself, or after its conversion into $\alpha-K G$, activates the mTORC1 pathway and participates in the growth signaling pathway. Evidence has shown that glutamine activates the mTORC1 pathway via Arf1 rather than via the Rag GTPase complex in MEFs ${ }^{190}$. According to another study, glutaminolysis increases the level of $\alpha-K G$ production, resulting in GTP loading of RagB and lysosomal translocation of the mTORC1 complex in human cancer cell lines ${ }^{160}$. It has been reported that cellular uptake of glutamine and its subsequent efflux in the presence of essential amino acids, including leucine, is the rate-determining step that activates $\mathrm{mTORC}^{191}$. Moreover, glutamine also acts as a precursor for the synthesis of various NEAAs, including asparagine and arginine, implicated in mTORC1 activation ${ }^{39}$. Thus, cells have diverse mechanisms of mTORC1 activation for glutamine, and cancer cells efficiently utilize glutamine for mTORC1 pathway activation to drive unrestrained oncogenic growth.

\section{Targeting glutamine metabolism and therapeutic implications}

Although the essential role of glutamine metabolism in cancer cells has been well demonstrated in vitro, the extent to which glutamine supports tumor growth and survival in vivo remains elusive. It has been reported that K-Ras-driven mouse lung tumors preferentially utilize glucose more than glutamine to supply carbon to the TCA cycle via pyruvate carboxylase ${ }^{192}$. Furthermore, human glioblastoma cells do not rely much on circulating glutamine for proliferation but rather more on glutamate to synthesize glutamine via glutamine synthetase to fuel purine biosynthesis ${ }^{193}$. Nevertheless, the specific metabolic importance of glutamine in tumorigenesis and tumor growth has also been reported ${ }^{194-196}$, and these studies have led many researchers to target glutamine metabolism for the treatment of cancer ${ }^{8}$. Throughout the discovery of agents targeting glutaminolysis, none have yet been used clinically ${ }^{197}$. A recent attempt focused on the inhibition of GLSs. GLS overexpression has been observed in different tumor cells, and these enzymes are found to function in the metabolic reprogramming of glutamine addiction in cancer ${ }^{198}$. Chemical agents targeting GLSs have been studied, and CB-839, 968, and BPTES have been found to exhibit tumor-specific antiproliferative effects ${ }^{199}$. Among these agents, CB-839 is the only one to proceed to clinical trials; however, its selectivity toward GLS1 and failure to inhibit the compensatory effect of GLS2 require in-depth study ${ }^{14}$. A recent study discovered a prodrug (JHU083) of the glutamine antagonist DON, which was designed to selectively become activated inside a tumor. The researchers showed that blocking glutamine metabolism through JHU083 not only suppressed tumor cell metabolism but also mitigated the tumor microenvironment, which is hostile to the immune response due to its hypoxic, acidic, and nutrientdepleted conditions, unleashing the natural antitumor $\mathrm{T}$ cell response. They also confirmed that concurrent treatment with JHU083 and anti-PD-1 checkpoint inhibitor improved the antitumor effects compared with antiPD-1 treatment alone, suggesting the presence of metabolic plasticity between cancer cells and effector $\mathrm{T}$ cells, which could be exploited as a metabolic checkpoint for cancer immunotherapy ${ }^{200}$.

The plasma membrane glutamine transporters SLC6A14, SLC7A11, and SLC38A1 have been targeted and found to be inhibited by erastin, $\alpha-M e-T r p$, and MeAIB, respectively (Fig. 8). In addition, SLC1A5 was shown to have clinical importance, and it is considered the most critical plasma membrane glutamine transporter in cancer cells ${ }^{201}$. Many attempts have been made to explore the possibility that SLC1A5 suppression via small molecules might exert anticancer effects. As part of this effort, benzylserine and benzylcysteine were discovered in 2004 as the first substrate analog inhibitors of SLC1A $5^{202}$. In an effort to improve the potency and efficacy of such inhibitors, some studies have discovered GPNA, which is widely used as a tool compound for suppressing SLC1A $5^{203}$. Other studies have developed antibodies with high affinity for SLC1A5, which induce antibodydependent cellular toxicity in gastric cancer models ${ }^{204}$. Recently, a potent inhibitor of SLC1A5, V-9302, has been reported to be effective in several cancer cell lines and in vivo tumor models ${ }^{205}$. However, other researchers have argued that controversial issues exist because GPNA also 


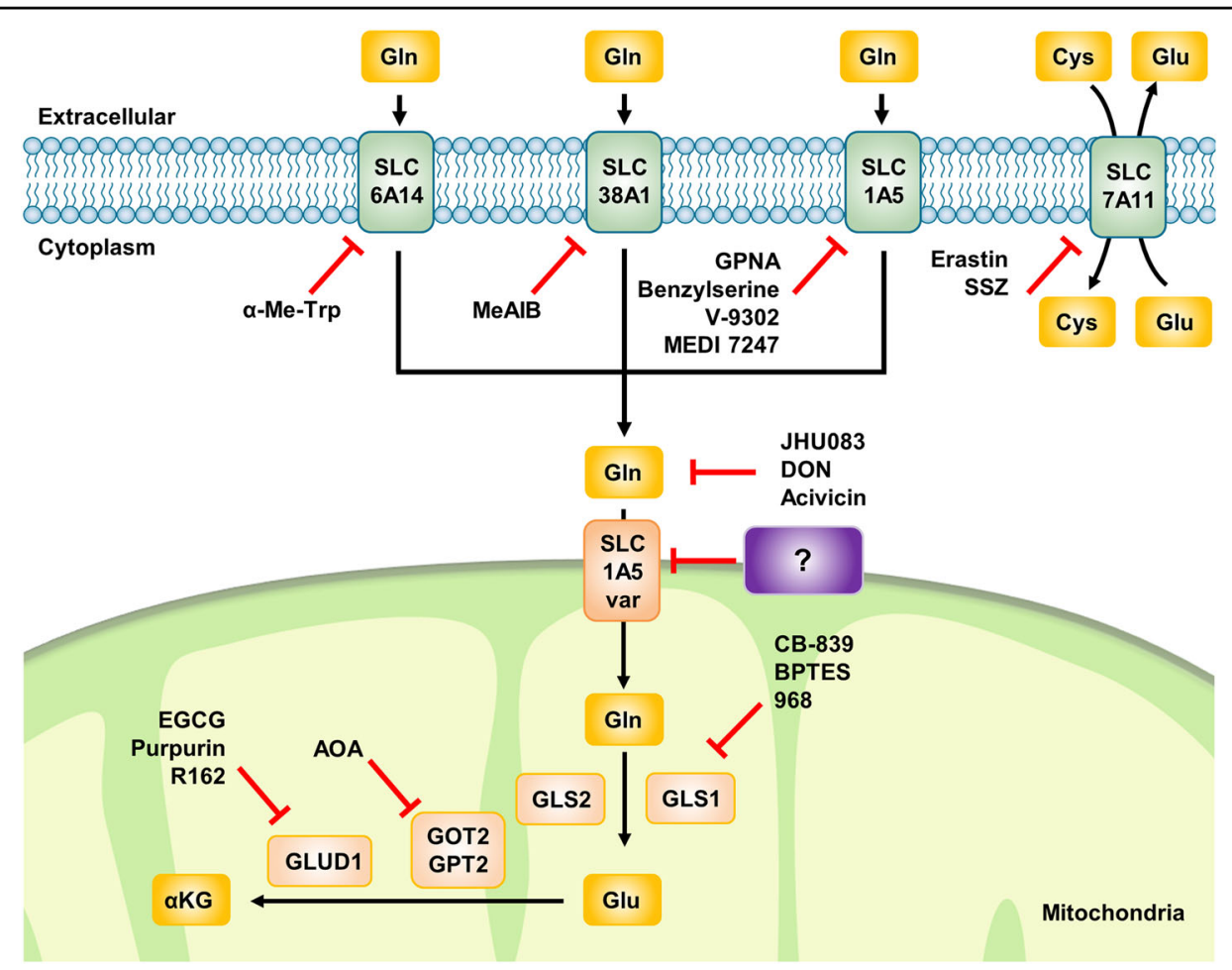

Fig. 8 Inhibitors of glutamine transporters and glutaminolysis. For the principal inhibition of glutaminolysis, attempts have been made to target the amino acid transporters related to these pathways. SLC6A14 and SLC38A1 are inhibited by a-Me-Trp and MelAB, respectively. The most intensely researched topic is inhibitors of SLC1A5, a major glutamine transporter, which include substrate analog competitive inhibitors such as GPNA, benzylserine, and V-9302 and the inhibitory antibody MEDI7247. Although they exhibit low potency, inhibitors of SLC7A11 include erastin and SSZ. Inhibitors of glutaminolytic enzymes are agents that target GLS1, GOT2, and GLUD1. CB-839, an agent in its 2nd clinical trial, inhibits GLS1 similarly to BPTES and 968. AOA inhibits GOT2 activity, and EGCG, purpurin, and R162 inactivate GLUD1. However, the SLC1A5 variant, the sole glutamine transporter discovered to date, is expected to be a much more effective target for cancer therapeutics than previously studied glutaminolysis inhibitors. Cys cysteine, Glu glutamate, a-KG a-ketoglutarate, GLS glutaminase, GOT2 glutamic-oxaloacetic transaminase 2, GPT2 glutamic-pyruvate transaminase 2, GLUD1 glutamate dehydrogenase 1, a-Me-Trp alpha-methyl-tryptophan, MeAIB methylaminoisobutyric acid, GPNA L-ү-glutamyl-pnitroanilide, SSZ sulfasalazine, DON 6-diazo-5-oxo-L-norleucine, AOA aminooxyacetate, EGCG epigallocatechin-3-gallate.

inhibits other glutamine transporters, such as SLC38A1, and V-9302 is effective even in SLC1A5 knockout mod$\mathrm{els}^{206,207}$. Hence, to date, no suitable compound has been identified to inhibit the plasma membrane glutamine transporter SLC1A5 with excellent sensitivity and specificity.

SLC1A5 might not be an appropriate target for suppressing glutamine uptake by cancer cells because it is not the only plasma membrane glutamine transporter, and its function would therefore be compensated by other redundant glutamine transporters such as SLC38A1 and SLC38A2. Thus, as the SLC1A5 variant is the only currently known glutamine transporter in the mitochondrial inner membrane ${ }^{16}$, targeting the SLC1A5 variant could be an effective strategy for selectively inhibiting glutamine metabolism in cancer cells (Fig. 8). Given the clinicopathological significance of SLC1A $5^{201}$ and the observation that the level of the SLC1A5 variant is negatively correlated with prognosis in several cancer types ${ }^{16}$, targeting the SLC1A5 variant is a promising strategy to starve cancer cells and induce antitumor effects. Therefore, further studies on the development of selective inhibitors of the mitochondrial SLC1A5 variant are needed and should help to establish whether the level of the SLC1A5 variant is a predictive marker of glutamine dependency in cancer ${ }^{21}$.

\section{Conclusion}

Although Otto Warburg characterized cancer metabolism by its enhanced glucose consumption and loss of mitochondrial function, many studies have shown that mitochondrial function in cancer cells is still robust and even enhanced. Moreover, glutamine has been discovered to be required for the maintenance of active mitochondrial function in cancer cells. Glutamine has historically been one of the most intensely investigated nutrients in cancer metabolism and is involved in various aspects of biosynthesis and bioenergetics, including NEAA production, epigenetic gene control, adaptation to hypoxic conditions, ATP synthesis, cell signaling, and tumorigenesis. 
In this review, we offer an updated overview of glutamine metabolism and discuss the reason for glutamine dependency in cell metabolism.

Certain types of cancer, including renal cell carcinoma, hematologic malignancies, glioblastoma, pancreatic cancer, and those reported to depend on HIF-2 $\alpha$, seem to depend on glutamine; hence, targeting glutamine metabolism may show therapeutic effects in these cancers. Moreover, metabolite transporters have recently been shown to be involved in tumorigenesis; for example, low levels of mitochondrial pyruvate carriers initiate colon cancer development ${ }^{208}$. Conversely, suppression of the SLC1A5 variant, a mitochondrial glutamine transporter, is sufficient to inhibit tumor growth by impairing glutamine metabolism in pancreatic cancer cells ${ }^{16}$. As the importance of subcellular metabolite transporters in controlling tumor initiation is poorly understood, it would be interesting to determine whether overexpression or knockout of these transporters is involved in tumorigenesis, metastasis, and immune modulation.

In conclusion, metabolic reliance on glutamine arises via the intrinsic functional diversity of glutamine, supporting macromolecule biosynthesis and reinforcing the TCA cycle. In the context of tumorigenesis, glutaminederived 2-HG alters the epigenetic landscape of chromosomes and induces oncogenic transformation. Further investigations to explain the mechanism underlying glutaminolysis-induced metabolic reprogramming are needed. These efforts are anticipated to reveal new metabolic vulnerabilities of cancer cells that can be targeted by therapeutic interventions.

\section{Acknowledgements}

This work was supported by the Basic Science Research Program through the National Research Foundation of Korea funded by the Ministry of Education (2018R1A6A1A03023718 and 2020R111A1A01067423) and by the NRF grant funded by the Korean government (MSIT) (2020M3E5E2040282). H.C.Y. was supported by the Graduate School of Yonsei University Research Scholarship Grants in 2019.

\section{Conflict of interest}

The authors declare that they have no conflict of interest.

\section{Publisher's note}

Springer Nature remains neutral with regard to jurisdictional claims in published maps and institutional affiliations.

Received: 7 May 2020 Revised: 22 July 2020 Accepted: 27 July 2020. Published online: 17 September 2020

\footnotetext{
References

1. Warburg, O. On the origin of cancer cells. Science 123, 309-314 (1956).

2. DeBerardinis, R. J. \& Cheng, T. Q's next: the diverse functions of glutamine in metabolism, cell biology and cancer. Oncogene 29, 313-324 (2010).

3. Hensley, C. T., Wasti, A. T. \& DeBerardinis, R. J. Glutamine and cancer: cell biology, physiology, and clinical opportunities. J. Clin. Invest. 123, 3678-3684 (2013).

4. Altman, B. J., Stine, Z. E. \& Dang, C. V. From Krebs to clinic: glutamine metabolism to cancer therapy. Nat. Rev. Cancer 16, 749 (2016).
}

5. Zhang, J., Pavlova, N. N. \& Thompson, C. B. Cancer cell metabolism: the essential role of the nonessential amino acid, glutamine. Embo J. 36 1302-1315 (2017).

6. Qing, G. et al. ATF4 regulates MYC-mediated neuroblastoma cell death upon glutamine deprivation. Cancer Cell 22, 631-644 (2012).

7. Chen, L. \& Cui, H. Targeting glutamine induces apoptosis: a cancer therapy approach. Int. J. Mol. Sci. 16, 22830-22855 (2015).

8. Wise, D. R. \& Thompson, C. B. Glutamine addiction: a new therapeutic target in cancer. Trends Biochem. Sci. 35, 427-433 (2010).

9. Klingman, J. D. \& Handler, P. Partial purification and properties of renal glutaminase. J. Biol. Chem. 232, 369-380 (1958).

10. Eagle, H. Nutrition needs of mammalian cells in tissue culture. Science $\mathbf{1 2 2}$ 501-514 (1955)

11. Kovacevic, Z. \& Morris, H. P. The role of glutamine in the oxidative metabolism of malignant cells. Cancer Res. 32, 326-333 (1972).

12. Reitzer, L. J., Wice, B. M. \& Kennell, D. Evidence that glutamine, not sugar, is the major energy source for cultured HeLa cells. J. Biol. Chem. 254, 2669-2676 (1979).

13. Lobo, C. et al. Inhibition of glutaminase expression by antisense mRNA decreases growth and tumourigenicity of tumour cells. Biochem. J. 348, 257-261 (2000).

14. Gross, M. I. et al. Antitumor activity of the glutaminase inhibitor CB-839 in triple-negative breast cancer. Mol. Cancer Ther. 13, 890-901 (2014).

15. Kizilbash, S. H. et al. The addition of CB-839 to temozolomide significantly reduces glioma aspartate and glutamate in an IDH mutated patient derived glioma xenograft model. Cancer Res. https://doi.org/10.1158/1538-7445. AM2019-3870 (2019).

16. Yoo, H. C. et al. A variant of SLC1A5 is a mitochondrial glutamine transporter for metabolic reprogramming in cancer cells. Cell Metab. 31, 267-283 (2020).

17. Scalise, M., Pochini, L., Galluccio, M., Console, L. \& Indiveri, C. Glutamine transport and mitochondrial metabolism in cancer cell growth. Front. Oncol. 7, 306 (2017).

18. Wang, J. B. et al. Targeting mitochondrial glutaminase activity inhibits oncogenic transformation. Cancer Cell 18, 397-397 (2010).

19. Li, B. et al. Targeting glutaminase 1 attenuates stemness properties in hepatocellular carcinoma by increasing reactive oxygen species and suppressing Wnt/beta-catenin pathway. EBioMedicine 39, 239-254 (2019).

20. Lukey, M. J. et al. Liver-type glutaminase GLS2 is a druggable metabolic node in luminal-subtype breast cancer. Cell Rep. 29, 76-88 e77 (2019).

21. Stine, Z. E. \& Dang, C. V. Glutamine skipping the Q into mitochondria. Trends Mol. Med. 26, 6-7 (2020).

22. Mullen, A. R. et al. Oxidation of alpha-ketoglutarate is required for reductive carboxylation in cancer cells with mitochondrial defects. Cell Rep. 7, 1679-1690 (2014).

23. Yang, L. F., Venneti, S. \& Nagrath, D. Glutaminolysis: a hallmark of cancer metabolism. Annu. Rev. Biomed. Eng. 19, 163-194 (2017).

24. Metallo, C. M. et al. Reductive glutamine metabolism by IDH1 mediates lipogenesis under hypoxia. Nature 481, 380-U166 (2012).

25. Sun, R. C. \& Denko, N. C. Hypoxic regulation of glutamine metabolism through HIF1 and SIAH2 supports lipid synthesis that is necessary for tumor growth. Cell Metab. 19, 285-292 (2014).

26. Lu, V. \& Teitell, M. A. Alpha-ketoglutarate: a "magic" metabolite in early germ cell development. Embo J. 38, e100615 (2019).

27. Chang, S., Yim, S. \& Park, H. The cancer driver genes IDH1/2, JARID1C/KDM5C, and UTX/KDM6A: crosstalk between histone demethylation and hypoxic reprogramming in cancer metabolism. Exp. Mol. Med. 51, 66 (2019).

28. Sullivan, L. B. et al. Supporting aspartate biosynthesis is an essential function of respiration in proliferating. Cell 162, 552-563 (2015).

29. Son, J. et al. Glutamine supports pancreatic cancer growth through a KRASregulated metabolic pathway. Nature https://doi.org/10.1038/nature12317 (2013).

30. Tong, X., Zhao, F. \& Thompson, C. B. The molecular determinants of de novo nucleotide biosynthesis in cancer cells. Curr. Opin. Genet. Dev. 19, 32-37 (2009).

31. Moffatt, B. A. \& Ashihara, H. Purine and pyrimidine nucleotide synthesis and metabolism. Arabidopsis Book 1, e0018 (2002).

32. Kim, J. et al. CPS1 maintains pyrimidine pools and DNA synthesis in KRAS/ LKB1-mutant lung cancer cells. Nature 546, 168-172 (2017).

33. Patel, D. et al. Aspartate rescues S-phase arrest caused by suppression of glutamine utilization in KRas-driven cancer cells. J. Biol. Chem. 291 9322-9329 (2016). 
34. Ben-Sahra, I., Howell, J. J., Asara, J. M. \& Manning, B. D. Stimulation of de novo pyrimidine synthesis by growth signaling through mTOR and S6K1. Science 339, 1323-1328 (2013)

35. Robitaille, A. M. et al. Quantitative phosphoproteomics reveal mTORC1 activates de novo pyrimidine synthesis. Science 339, 1320-1323 (2013).

36. Liu, Y. C. et al. Global regulation of nucleotide biosynthetic genes by c-Myc PLOS ONE 3, e2722 (2008).

37. Lane, A. N. \& Fan, T. W. Regulation of mammalian nucleotide metabolism and biosynthesis. Nucleic Acids Res. 43, 2466-2485 (2015).

38. Santana-Codina, N. et al. Oncogenic KRAS supports pancreatic cancer through regulation of nucleotide synthesis. Nat. Commun. 9, 4945 (2018).

39. Choi, B. H. \& Coloff, J. L. The diverse functions of non-essential amino acids in cancer. Cancers 11, 675 (2019).

40. Thornburg, J. M. et al. Targeting aspartate aminotransferase in breast cancer Breast Cancer Res. 10, 1-12 (2008).

41. Korangath, P. et al. Targeting glutamine metabolism in breast cancer with aminooxyacetate. Clin. Cancer Res. 21, 3263-3273 (2015).

42. Liu, B. Y. et al. Overexpression of phosphoserine aminotransferase 1 (PSAT1) predicts poor prognosis and associates with tumor progression in human esophageal squamous cell carcinoma. Cell. Physiol. Biochem. 39, 395-406 (2016).

43. Zhang, J. et al. Asparagine plays a critical role in regulating cellular adaptation to glutamine depletion. Mol. Cell 56, 205-218 (2014).

44. Huang, $\mathrm{H}$. et al. Role of glutamine and interlinked asparagine metabolism in vessel formation. Embo J. 36, 2334-2352 (2017).

45. Pavlova, N. N. et al. As extracellular glutamine levels decline, asparagine becomes an essential amino acid. Cell Metab. 27, 428 (2018).

46. LeBoeuf, S. E. et al. Activation of oxidative stress response in cancer generates a druggable dependency on exogenous non-essential amino acids. Cell Metab. 31, 339 (2020).

47. Sayin, V. I. et al. Activation of the NRF2 antioxidant program generates an imbalance in central carbon metabolism in cancer. elife 6, e28083 (2017).

48. Coloff, J. L. et al. Differential glutamate metabolism in proliferating and quiescent mammary epithelial cells. Cell Metab. 23, 867-880 (2016).

49. Zhu, Y. et al. A critical role of glutamine and asparagine gamma-nitrogen in nucleotide biosynthesis in cancer cells hijacked by an oncogenic virus. Mbio 8, e01179-17 (2017).

50. Pant, A., Cao, S. \& Yang, Z. L. Asparagine is a critical limiting metabolite for vaccinia virus protein synthesis during glutamine deprivation. J. Virol. 93, e01834-18 (2019).

51. Knott, S. R. V. et al. Asparagine bioavailability governs metastasis in a model of breast cancer. Nature https://doi.org/10.1038/nature26162 (2018).

52. Hill, J. M. et al. L-asparaginase therapy for leukemia and other malignant neoplasms. Remission in human leukemia. Jama 202, 882-888 (1967).

53. Krall, A. S., Xu, S., Graeber, T. G., Braas, D. \& Christofk, H. R. Asparagine promotes cancer cell proliferation through use as an amino acid exchange factor. Nat. Commun. 7, 11457 (2016).

54. Krall, A. S. et al. Asparagine signals mitochondrial respiration and can be targeted to impair tumour growth. bioRxiv, 2020.2003.2017.995670 (2020).

55. Weinberg, F. et al. Mitochondrial metabolism and ROS generation are essential for Kras-mediated tumorigenicity. Proc. Natl Acad. Sci. USA 107 8788-8793 (2010).

56. Gorrini, C., Harris, I. S. \& Mak, T. W. Modulation of oxidative stress as an anticancer strategy. Nat. Rev. Drug Discov. 12, 931-947 (2013).

57. Amores-Sanchez, M. I. \& Medina, M. A. Glutamine, as a precursor of glutathione, and oxidative stress. Mol. Genet. Metab. 67, 100-105 (1999).

58. Welbourne, T. C. Ammonia production and glutamine incorporation into glutathione in the functioning rat kidney. Can. J. Biochem. 57, 233-237 (1979).

59. Sappington, D. R. et al. Glutamine drives glutathione synthesis and contributes to radiation sensitivity of A549 and H460 lung cancer cell lines. Biochim. Biophys. Acta 1860, 836-843 (2016).

60. Tompkins, S. C. et al. Disrupting mitochondrial pyruvate uptake directs glutamine into the TCA cycle away from glutathione synthesis and impairs hepatocellular tumorigenesis. Cell Rep. 28, 2608-2619 (2019).

61. Muir, A. et al. Environmental cystine drives glutamine anaplerosis and sensitizes cancer cells to glutaminase inhibition. elife https://doi.org/10.7554/ elife.27713 (2017).

62. Timmerman, L. A. et al. Glutamine sensitivity analysis identifies the $x C T$ antiporter as a common triple-negative breast tumor therapeutic target. Cancer Cell 24, 450-465 (2013).
63. Okazaki, K., Papagiannakopoulos, T. \& Motohashi, H. Metabolic features of cancer cells in NRF2 addiction status. Biophys. Rev. https://doi.org/10.1007/ s12551-020-00659-8 (2020).

64. Jiang, L. et al. Reductive carboxylation supports redox homeostasis during anchorage-independent growth. Nature 532, 255-258 (2016).

65. Morotti, M. et al. Hypoxia-induced switch in SNAT2/SLC38A2 regulation generates endocrine resistance in breast cancer. Proc. Natl Acad. Sci. USA 116 12452-12461 (2019).

66. Kim, J. W., Tchernyshyov, I., Semenza, G. L. \& Dang, C. V. HIF-1-mediated expression of pyruvate dehydrogenase kinase: a metabolic switch required for cellular adaptation to hypoxia. Cell Metab. 3, 177-185 (2006).

67. Denko, N. C. Hypoxia, HIF1 and glucose metabolism in the solid tumour. Nat. Rev. Cancer 8, 705-713 (2008).

68. Goda, N. \& Kanai, M. Hypoxia-inducible factors and their roles in energy metabolism. Int. J. Hematol. 95, 457-463 (2012)

69. Zhao, J., Du, F., Shen, G., Zheng, F. \& Xu, B. The role of hypoxia-inducible factor-2 in digestive system cancers. Cell Death Dis. 6, e1600 (2015).

70. Li, Z. et al. Hypoxia-inducible factors regulate tumorigenic capacity of glioma stem cells. Cancer Cell 15, 501-513 (2009).

71. Corbet, $\mathrm{C}$. et al. The SIRT1/HIF2alpha axis drives reductive glutamine metabolism under chronic acidosis and alters tumor response to therapy. Cancer Res. 74, 5507-5519 (2014).

72. Perez-Escuredo, J. et al. Lactate promotes glutamine uptake and metabolism in oxidative cancer cells. Cell Cycle 15, 72-83 (2016).

73. Currie, E., Schulze, A., Zechner, R., Walther, T. C. \& Farese, R. V. Jr. Cellular fatty acid metabolism and cancer. Cell Metab. 18, 153-161 (2013).

74. Wise, D. R. et al. Hypoxia promotes isocitrate dehydrogenase-dependent carboxylation of alpha-ketoglutarate to citrate to support cell growth and viability. Proc. Natl Acad. Sci. USA 108, 19611-19616 (2011).

75. Metallo, C. M. et al. Reductive glutamine metabolism by IDH1 mediates lipogenesis under hypoxia. Nature 481, 380-384 (2011).

76. Mullen, A. R. et al. Reductive carboxylation supports growth in tumour cells with defective mitochondria. Nature 481, 385-388 (2011).

77. Qiu, B. et al. HIF2alpha-dependent lipid storage promotes endoplasmic reticulum homeostasis in clear-cell renal cell carcinoma. Cancer Discov. 5, 652-667 (2015).

78. Du, W. et al. HIF drives lipid deposition and cancer in CCRCC via repression of fatty acid metabolism. Nat. Commun. 8, 1769 (2017).

79. Scheuermann, T. H. et al. Allosteric inhibition of hypoxia inducible factor-2 with small molecules. Nat. Chem. Biol. 9, 271-276 (2013).

80. Wallace, E. M. et al. A small-molecule antagonist of HIF2alpha Is efficacious in preclinical models of renal cell carcinoma. Cancer Res. 76, 5491-5500 (2016).

81. Chen, W. et al. Targeting renal cell carcinoma with a HIF-2 antagonist. Nature 539, 112-117 (2016)

82. Cho, $\mathrm{H}$. et al. On-target efficacy of a HIF-2alpha antagonist in preclinical kidney cancer models. Nature 539, 107-111 (2016).

83. Wappler, J. et al. Glutamine deprivation counteracts hypoxia-induced chemoresistance. Neoplasia 22, 22-32 (2020).

84. Chen, $R$. et al. Disrupting glutamine metabolic pathways to sensitize gemcitabine-resistant pancreatic cancer. Sci. Rep. 7, 7950 (2017).

85. Mukhopadhyay, S. et al. Undermining glutaminolysis bolsters chemotherapy while NRF2 promotes chemoresistance in KRAS-driven pancreatic cancers. Cancer Res. https://doi.org/10.1158/0008-5472.CAN-19-1363 (2020).

86. Ju, H. Q. et al. Mechanisms of overcoming intrinsic resistance to gemcitabine in pancreatic ductal adenocarcinoma through the redox modulation. Mol. Cancer Ther. 14, 788-798 (2015).

87. Shukla, S. K. et al. MUC1 and HIF-1alpha signaling crosstalk induces anabolic glucose metabolism to impart gemcitabine resistance to pancreatic cancer. Cancer Cell 32, 392 (2017).

88. Intlekofer, A. M. et al. Hypoxia induces production of L-2-hydroxyglutarate Cell Metab. 22, 304-311 (2015).

89. Oldham, W. M., Clish, C. B., Yang, Y. \& Loscalzo, J. Hypoxia-mediated increases in $\mathrm{L}$-2-hydroxyglutarate coordinate the metabolic response to reductive stress. Cell Metab. 22, 291-303 (2015).

90. Nadtochiy, S. M. et al. Acidic pH is a metabolic switch for 2-hydroxyglutarate generation and signaling. J. Biol. Chem. 291, 20188-20197 (2016).

91. Intlekofer, A. M. et al. L-2-hydroxyglutarate production arises from noncanonical enzyme function at acidic pH. Nat. Chem. Biol. 13, 494-500 (2017)

92. Kranendijk, M., Struys, E. A., Salomons, G. S., Van der Knaap, M. S. \& Jakobs, C. Progress in understanding 2-hydroxyglutaric acidurias. J. Inherit. Metab. Dis. 35, 571-587 (2012). 
93. Aghili, M., Zahedi, F. \& Rafiee, E. Hydroxyglutaric aciduria and malignant brain tumor: a case report and literature review. J. NeuroOncol. 91, 233-236 (2009).

94. Larnaout, A. et al. Osteoma of the calvaria in L-2-hydroxyglutaric aciduria. J. Inherit. Metab. Dis. 30, 980 (2007).

95. Rogers, R. E. et al. Wilms tumor in a child with L-2-hydroxyglutaric aciduria. Pediatr. Dev. Pathol. 13, 408-411 (2010).

96. Shim, E. H. et al. L-2-Hydroxyglutarate: an epigenetic modifier and putative oncometabolite in renal cancer. Cancer Discov. 4, 1290-1298 (2014).

97. Reid, M. A., Dai, Z. W. \& Locasale, J. W. The impact of cellular metabolism on chromatin dynamics and epigenetics. Nat. Cell Biol. 19, 1298-1306 (2017).

98. Schvartzman, J. M., Thompson, C. B. \& Finley, L. W. S. Metabolic regulation of chromatin modifications and gene expression. J. Cell Biol. 217, 2247-2259 (2018).

99. Astuti, D. et al. Gene mutations in the succinate dehydrogenase subunit SDHB cause susceptibility to familial pheochromocytoma and to familial paraganglioma. Am. J. Hum. Genet. 69, 640-640 (2001).

100. Letouze, E. et al. SDH mutations establish a hypermethylator phenotype in paraganglioma. Cancer Cell 23, 739-752 (2013).

101. Janeway, K. A. et al. Defects in succinate dehydrogenase in gastrointestinal stromal tumors lacking KIT and PDGFRA mutations. Proc. Natl Acad. Sci. USA 108, 314-318 (2011).

102. Killian, J. K. et al. Succinate dehydrogenase mutation underlies global epigenomic divergence in gastrointestinal stromal tumor. Cancer Discov. 3 648-657 (2013).

103. Pan, M. et al. Regional glutamine deficiency in tumours promotes dedifferentiation through inhibition of histone demethylation. Nat. Cell Biol. 18, 1090-1101 (2016).

104. Carey, B. W., Finley, L. W., Cross, J. R., Allis, C. D. \& Thompson, C. B. Intracellular alpha-ketoglutarate maintains the pluripotency of embryonic stem cells. Nature 518, 413-416 (2015)

105. TeSlaa, T. et al. alpha-Ketoglutarate accelerates the initial differentiation of primed human pluripotent stem cells. Cell Metab. 24, 485-493 (2016).

106. Hwang, I. Y. et al. Psat1-dependent fluctuations in alpha-ketoglutarate affect the timing of ESC differentiation. Cell Metab. 24, 494-501 (2016).

107. Yu, Y. et al. Glutamine metabolism regulates proliferation and lineage allocation in skeletal stem cells. Cell Metab. 29, 966-978 (2019).

108. Liu, P. S. et al. alpha-ketoglutarate orchestrates macrophage activation through metabolic and epigenetic reprogramming. Nat. Immunol. 18, 985 (2017).

109. Klysz, D. et al. Glutamine-dependent alpha-ketoglutarate production regulates the balance between T helper 1 cell and regulatory $T$ cell generation. Sci. Signal. 8, ra97 (2015).

110. Petrus, P. et al. Glutamine links obesity to inflammation in human white adipose tissue. Cell Metab. 31, 375 (2020).

111. Dang, L. et al. Cancer-associated IDH1 mutations produce 2hydroxyglutarate. Nature 462, 739-744 (2009).

112. Martinez-Reyes, I. \& Chandel, N. S. Mitochondrial TCA cycle metabolites control physiology and disease. Nat. Commun. 11, 102 (2020).

113. Ward, P. S. et al. The common feature of leukemia-associated IDH1 and IDH2 mutations is a neomorphic enzyme activity converting alpha-ketoglutarate to 2-hydroxyglutarate. Cancer Cell 17, 225-234 (2010).

114. Amary, M. F. et al. IDH1 and IDH2 mutations are frequent events in central chondrosarcoma and central and periosteal chondromas but not in other mesenchymal tumours. J. Pathol. 224, 334-343 (2011).

115. Borger, D. R. et al. Circulating oncometabolite 2-hydroxyglutarate is a potential surrogate biomarker in patients with isocitrate dehydrogenasemutant intrahepatic cholangiocarcinoma. Clin. Cancer Res. 20, 1884-1890 (2014)

116. Losman, J. A. et al. (R)-2-hydroxyglutarate is sufficient to promote leukemogenesis and its effects are reversible. Science 339, 1621-1625 (2013).

117. Figueroa, M. E. et al. Leukemic IDH1 and IDH2 mutations result in a hypermethylation phenotype, disrupt TET2 function, and impair hematopoietic differentiation. Cancer Cell 18, 553-567 (2010).

118. Lu, C. et al. IDH mutation impairs histone demethylation and results in a block to cell differentiation. Nature 483, 474-478 (2012).

119. Ohka, F. et al. Quantitative metabolome analysis profiles activation of glutaminolysis in glioma with IDH1 mutation. Tumour Biol. 35, 5911-5920 (2014).

120. Tateishi, $\mathrm{K}$. et al. Extreme vulnerability of IDH1 mutant cancers to NAD+ depletion. Cancer Cell 28, 773-784 (2015).

121. Salamanca-Cardona, $L$. et al. In vivo imaging of glutamine metabolism to the oncometabolite 2-hydroxyglutarate in IDH1/2 mutant tumors. Cell Metab. 26 830-841 e833 (2017).
122. Gonsalves, W. I. et al. Glutamine-derived 2-hydroxyglutarate is associated with disease progression in plasma cell malignancies. JCl Insight https://doi.org/ 10.1172/jci.insight.94543 (2018).

123. Matre, P. et al. Inhibiting glutaminase in acute myeloid leukemia: metabolic dependency of selected AML subtypes. Oncotarget 7, 79722-79735 (2016).

124. Richter, S. et al. Krebs cycle metabolite profiling for identification and stratification of pheochromocytomas/paragangliomas due to succinate dehydrogenase deficiency. J. Clin. Endocrinol. Metab. 99, 3903-3911 (2014).

125. Bardella, C., Pollard, P. J. \& Tomlinson, I. SDH mutations in cancer. Biochim Biophys. Acta 1807, 1432-1443 (2011).

126. Müller, S. N. U. Mutations in SDHC cause autosomal dominant paraganglioma, type 3. Nat. Genet. 26, 268-270 (2000).

127. Selak, M. A. et al. Succinate links TCA cycle dysfunction to oncogenesis by inhibiting HIF-alpha prolyl hydroxylase. Cancer Cell 7, 77-85 (2005).

128. Astuti, D. et al. Gene mutations in the succinate dehydrogenase subunit SDHB cause susceptibility to familial pheochromocytoma and to familial paraganglioma. Am. J. Hum. Genet. 69, 49-54 (2001).

129. Virpi Launonen, O. V. et al. Inherited susceptibility to uterine leiomyomas and renal cell cancer. PNAS 98, 3387-3392 (2001)

130. Tomlinson, I. P. et al. Germline mutations in $\mathrm{FH}$ predispose to dominantly inherited uterine fibroids, skin leiomyomata and papillary renal cell cancer. Nat. Genet. 30, 406-410 (2002).

131. Sciacovelli, M. et al. Fumarate is an epigenetic modifier that elicits epithelialto-mesenchymal transition. Nature 537, 544-547 (2016).

132. Laukka, T. et al. Fumarate and succinate regulate expression of hypoxiainducible genes via TET enzymes. J. Biol. Chem. 291, 4256-4265 (2016).

133. Isaacs, J. S. et al. HIF overexpression correlates with biallelic loss of fumarate hydratase in renal cancer: novel role of fumarate in regulation of HIF stability. Cancer Cell 8, 143-153 (2005).

134. Kinch, L., Grishin, N. V. \& Brugarolas, J. Succination of Keap1 and activation of $\mathrm{Nrf2}$-dependent antioxidant pathways in $\mathrm{FH}$-deficient papillary renal cell carcinoma type 2. Cancer Cell 20, 418-420 (2011).

135. Adam, J. et al. Renal cyst formation in Fh1-deficient mice is independent of the Hif/Phd pathway: roles for fumarate in KEAP1 succination and Nrf2 signaling. Cancer Cell 20, 524-537 (2011).

136. Sullivan, L. B. et al. The proto-oncometabolite fumarate binds glutathione to amplify ROS-dependent signaling. Mol. Cell 51, 236-248 (2013).

137. Ooi, A. et al. An antioxidant response phenotype shared between hereditary and sporadic type 2 papillary renal cell carcinoma. Cancer Cell 20, 511-523 (2011).

138. Ternette, $\mathrm{N}$. et al. Inhibition of mitochondrial aconitase by succination in fumarate hydratase deficiency. Cell Rep. 3, 689-700 (2013).

139. Greenhouse, W. V. \& Lehninger, A. L. Magnitude of malate-aspartate reduced nicotinamide adenine dinucleotide shuttle activity in intact respiring tumor cells. Cancer Res. 37, 4173-4181 (1977).

140. Fan, J. et al. Glutamine-driven oxidative phosphorylation is a major ATP source in transformed mammalian cells in both normoxia and hypoxia. Mol. Syst. Biol. 9, 712 (2013).

141. Carracedo, A., Cantley, L. C. \& Pandolfi, P. P. Cancer metabolism: fatty acid oxidation in the limelight. Nat. Rev. Cancer 13, 227-232 (2013).

142. Kang, J. H. et al. Aldehyde dehydrogenase inhibition combined with phenformin treatment reversed NSCLC through ATP depletion. Oncotarget 7 49397-49410 (2016)

143. Lee, J. S. et al. Dual targeting of glutaminase 1 and thymidylate synthase elicits death synergistically in NSCLC. Cell Death Dis. 7, e2511 (2016).

144. Andrews, F. J. \& Griffiths, R. D. Glutamine: essential for immune nutrition in the critically ill. Br. J. Nutr. 87, S3-S8 (2002).

145. Ferreira, C. et al. Glutamine supplementation improves the efficacy of miltefosine treatment for visceral leishmaniasis. PLoS Negl. Trop. Dis. 14, e0008125 (2020)

146. Gianotti, L., Alexander, J. W., Gennari, R., Pyles, T. \& Babcock, G. F. Oral glutamine decreases bacterial translocation and improves survival in experimental gut-origin sepsis. J. Parenter. Enter. Nutr. 19, 69-74 (1995).

147. Bakalar, B. et al. Parenterally administered dipeptide alanyl-glutamine prevents worsening of insulin sensitivity in multiple-trauma patients. Crit. Care Med. 34, 381-386 (2006).

148. Reid, M. A. et al. The B55alpha subunit of PP2A drives a p53-dependent metabolic adaptation to glutamine deprivation. Mol. Cell 50, 200-211 (2013).

149. Tajan, M. et al. A role for p53 in the adaptation to glutamine starvation through the expression of SLC1A3. Cell Metab. 28, 721 (2018). 
150. Fu, S. J. et al. Glutamine synthetase promotes radiation resistance via facilitating nucleotide metabolism and subsequent DNA damage repair. Cell Rep. 28, 1136 (2019).

151. Jeong, S. M. et al. SIRT4 has tumor-suppressive activity and regulates the cellular metabolic response to DNA damage by inhibiting mitochondrial glutamine metabolism. Cancer Cell 23, 450-463 (2013).

152. Kim, M., Gwak, J., Hwang, S., Yang, S. \& Jeong, S. M. Mitochondrial GPT2 plays a pivotal role in metabolic adaptation to the perturbation of mitochondrial glutamine metabolism. Oncogene 38, 4729-4738 (2019).

153. Cheng, T. et al. Pyruvate carboxylase is required for glutamine-independent growth of tumor cells. Proc. Natl Acad. Sci. USA 108, 8674-8679 (2011).

154. Jitrapakdee, S., Vidal-Puig, A. \& Wallace, J. C. Anaplerotic roles of pyruvate carboxylase in mammalian tissues. Cell. Mol. Life Sci. 63, 843-854 (2006).

155. Christen, S. et al. Breast cancer-derived lung metastases show increased pyruvate carboxylase-dependent anaplerosis. Cell Rep. 17, 837-848 (2016).

156. Cappel, D. A. et al. Pyruvate-carboxylase-mediated anaplerosis promotes antioxidant capacity by sustaining TCA cycle and redox metabolism in liver. Cell Metab. 29, 1291-1305 (2019).

157. Szeliga, M. \& Obara-Michlewska, M. Glutamine in neoplastic cells: focus on the expression and roles of glutaminases. Neurochem. Int. 55, 71-75 (2009).

158. Eng, C. H. \& Abraham, R. T. Glutaminolysis yields a metabolic by-product that stimulates autophagy. Autophagy 6, 968-970 (2010).

159. Rabinowitz, J. D. \& White, E. Autophagy and metabolism. Science $\mathbf{3 3 0}$ 1344-1348 (2010).

160. Duran, R. V. \& Hall, M. N. Glutaminolysis feeds mTORC1. Cell Cycle 11, 4107-4108 (2012).

161. Duran, R. V. et al. HIF-independent role of prolyl hydroxylases in the cellular response to amino acids. Oncogene 32, 4549-4556 (2013).

162. Poulain, L. et al. High mTORC1 activity drives glycolysis addiction and sensitivity to G6PD inhibition in acute myeloid leukemia cells. Leukemia $\mathbf{3 1}$ 2326-2335 (2017).

163. Dutchak, P. A. et al. Loss of a negative regulator of mTORC1 induces aerobic glycolysis and altered fiber composition in skeletal muscle. Cell Rep. 23, 1907-1914 (2018).

164. Sanli, T., Steinberg, G. R., Singh, G. \& Tsakiridis, T. AMP-activated protein kinase (AMPK) beyond metabolism: a novel genomic stress sensor participating in the DNA damage response pathway. Cancer Biol. Ther. 15, 156-169 (2014).

165. Shen, C. \& Houghton, P. J. The mTOR pathway negatively controls ATM by upregulating miRNAs. Proc. Natl Acad. Sci. USA 110, 11869-11874 (2013).

166. Villar, V. H., Merhi, F., Djavaheri-Mergny, M. \& Duran, R. V. Glutaminolysis and autophagy in cancer. Autophagy 11, 1198-1208 (2015).

167. Eng, C. H., Yu, K., Lucas, J., White, E. \& Abraham, R. T. Ammonia derived from glutaminolysis is a diffusible regulator of autophagy. Sci. Signal. 3, ra31 (2010).

168. Medvetz, D., Priolo, C. \& Henske, E. P. Therapeutic targeting of cellular metabolism in cells with hyperactive MTORC1: a paradigm shift. Mol. Cancer Res. 13, 3-8 (2015)

169. Debnath, J. Detachment-induced autophagy during anoikis and lumen formation in epithelial acini. Autophagy 4, 351-353 (2008).

170. Fan, Q. et al. Autophagy promotes metastasis and glycolysis by upregulating MCT1 expression and Wnt/beta-catenin signaling pathway activation in hepatocellular carcinoma cells. J. Exp. Clin. Cancer Res. 37, 9 (2018).

171. Soria, L. R. \& Brunetti-Pierri, N. Ammonia and autophagy: An emerging relationship with implications for disorders with hyperammonemia. J. Inherit. Metab. Dis. 42, 1097-1104 (2019).

172. Marino, G. \& Kroemer, G. Ammonia: a diffusible factor released by proliferating cells that induces autophagy. Sci. Signal. 3, pe19 (2010).

173. Vander Heiden, M. G., Cantley, L. C. \& Thompson, C. B. Understanding the Warburg effect: the metabolic requirements of cell proliferation. Science $\mathbf{3 2 4}$, 1029-1033 (2009).

174. Dang, C. V. Rethinking the Warburg effect with Myc micromanaging glutamine metabolism. Cancer Res. 70, 859-862 (2010).

175. Hao, Y. et al. Oncogenic PIK3CA mutations reprogram glutamine metabolism in colorectal cancer. Nat. Commun. 7, 11971 (2016).

176. Stine, Z. E., Walton, Z. E., Altman, B. J., Hsieh, A. L. \& Dang, C. V. MYC, metabolism, and cancer. Cancer Discov. 5, 1024-1039 (2015).

177. Wise, D. R. et al. Myc regulates a transcriptional program that stimulates mitochondrial glutaminolysis and leads to glutamine addiction. Proc. Natl Acad. Sci. USA 105, 18782-18787 (2008).

178. Gao, P. et al. c-Myc suppression of miR-23a/b enhances mitochondrial glutaminase expression and glutamine metabolism. Nature 458, 762-765 (2009).
179. Yuneva, M. O. et al. The metabolic profile of tumors depends on both the responsible genetic lesion and tissue type. Cell Metab. 15, 157-170 (2012).

180. Xiao, D. et al. Myc promotes glutaminolysis in human neuroblastoma through direct activation of glutaminase 2. Oncotarget 6, 40655-40666 (2015).

181. Toda, K. et al. Clinical role of ASCT2 (SLC1A5) in KRAS-mutated colorectal cancer. Int. J. Mol. Sci. https://doi.org/10.3390/ijms18081632 (2017).

182. Bernfeld, E. \& Foster, D. A. Glutamine as an essential amino acid for KRasdriven cancer cells. Trends Endocrinol. Metab. 30, 357-368 (2019).

183. Anglin, J. et al. Discovery and optimization of aspartate aminotransferase 1 inhibitors to target redox balance in pancreatic ductal adenocarcinoma. Bioorg. Med. Chem. Lett. 28, 2675-2678 (2018).

184. Patel, D. et al. Aspartate rescues S-phase arrest caused by suppression of glutamine utilization in KRas-driven cancer cells. J. Biol. Chem. 291 9322-9329 (2016).

185. Saqcena, M. et al. Blocking anaplerotic entry of glutamine into the TCA cycle sensitizes K-Ras mutant cancer cells to cytotoxic drugs. Oncogene $\mathbf{3 4}$, 2672-2680 (2015).

186. Saqcena, M. et al. Amino acids and mTOR mediate distinct metabolic checkpoints in mammalian G1 cell cycle. PLOS ONE 8, e74157 (2013).

187. Gwinn, D. M. et al. Oncogenic KRAS regulates amino acid homeostasis and asparagine biosynthesis via ATF4 and alters sensitivity to L-asparaginase. Cancer Cell 33, 91-107 (2018).

188. Mei, Z. B., Duan, C. Y., Li, C. B., Cui, L. \& Ogino, S. Prognostic role of tumor PIK3CA mutation in colorectal cancer: a systematic review and meta-analysis. Ann. Oncol. 27, 1836-1848 (2016).

189. Csibi, A. et al. The mTORC1 pathway stimulates glutamine metabolism and cell proliferation by repressing SIRT4. Cell 153, 840-854 (2013).

190. Jewell, J. L. et al. Differential regulation of mTORC1 by leucine and glutamine. Science 347, 194-198 (2015).

191. Nicklin, P. et al. Bidirectional transport of amino acids regulates mTOR and autophagy. Cell 136, 521-534 (2009).

192. Davidson, S. M. et al. Environment impacts the metabolic dependencies of Ras-driven non-small cell lung cancer. Cell Metab. 23, 517-528 (2016).

193. Tardito, S. et al. Glutamine synthetase activity fuels nucleotide biosynthesis and supports growth of glutamine-restricted glioblastoma. Nat. Cell Biol. 17, 1556-1568 (2015).

194. Le, A. et al. Glucose-independent glutamine metabolism via TCA cycling for proliferation and survival in B cells. Cell Metab. 15, 110-121 (2012).

195. Xiang, Y. et al. Targeted inhibition of tumor-specific glutaminase diminishes cell-autonomous tumorigenesis. J. Clin. Invest. 125, 2293-2306 (2015).

196. Shroff, E. H. et al. MYC oncogene overexpression drives renal cell carcinoma in a mouse model through glutamine metabolism. Proc. Natl Acad. Sci. USA 112, 6539-6544 (2015)

197. jin, L., A., G. N. \& Kang., S. Glutaminolysis as a target for cancer therapy. Oncogene 35, 3619-3625 (2016).

198. Lobo, C. et al. Inhibition of glutaminase expression by antisense mRNA decreases growth and tumourigenicity of tumour cells. Biochem. J. 348, 257-261 (2000).

199. Hoerner, C. R., Chen, V. J. \& Fan, A. C. The 'Achilles Heel' of metabolism in renal cell carcinoma: glutaminase inhibition as a rational treatment strategy. Kidney Cancer 3, 15-29 (2019).

200. Leone, R. D. et al. Glutamine blockade induces divergent metabolic programs to overcome tumor immune evasion. Science 366, 1013 (2019).

201. Liu, Y. et al. The role of ASCT2 in cancer: a review. Eur. J. Pharm. 837, 81-87 (2018).

202. Grewer, C. \& G., E. New inhibitors for the neutral amino acid transporter ASCT2 reveal its Na+-dependent anion leak. J. Physiol. 557, 747-759 (2004).

203. Esslinger, C. S., Cybulski, K. A. \& Rhoderick, J. F. Ngamma-aryl glutamine analogues as probes of the ASCT2 neutral amino acid transporter binding site. Bioorg. Med. Chem. 13, 1111-1118 (2005).

204. Osanai-Sasakawa, A. et al. An anti-ASCT2 monoclonal antibody suppresses gastric cancer growth by inducing oxidative stress and antibody dependent cellular toxicity in preclinical models. Am. J. Cancer Res. 8, 1499-1513 (2018).

205. Schulte, M. L. et al. Pharmacological blockade of ASCT2-dependent glutamine transport leads to antitumor efficacy in preclinical models. Nat. Med. 24, 194-202 (2018).

206. Broer, A. Fairweather, S. \& Broer, S. Disruption of amino acid homeostasis by novel ASCT2 inhibitors involves multiple targets. Front. Pharm. 9, 785 (2018).

207. Chiu, M. et al. GPNA inhibits the sodium-independent transport system $L$ for neutral amino acids. Amino Acids 49, 1365-1372 (2017).

208. Bensard, C. L. et al. Regulation of tumor initiation by the mitochondrial pyruvate carrier. Cell Metab. 31, 284-300 (2020). 